\title{
A Novel Synthesis of Isonucleosides
}

\author{
Yuichi Yoshimura, ${ }^{1, *}$ Kazuhiro Asami, ${ }^{1}$ Hiromitsu Matsui, ${ }^{2}$ \\ Hiromichi Tanaka, ${ }^{2}$ and Hiroki Takahata ${ }^{1, *}$
}

${ }^{1}$ Tohoku Pharmaceutical University, 4-4-1 Komatsushima, Aoba-ku, Sendai 981-8558, Japan; ${ }^{2}$ School of Pharmaceutical Sciences, Showa University, 1-5-8 Hatanodai, Shinagawa-ku, Tokyo 142-8555, Japan

\section{Supporting Information}

\section{Contents}

Experimental Procedures and Characterization Data S-2

${ }^{1} \mathrm{H}$ and ${ }^{13} \mathrm{C}$ NMR Spectra $\quad \mathrm{S}-10$ 


\section{Experimental Procedures and Characterization Data}

All the reactions described were performed under argon atmosphere unless any conditions were described. Melting points are uncorrected. NMR spectra were recorded at $400 \mathrm{MHz}\left({ }^{1} \mathrm{H}\right)$ and $100 \mathrm{MHz}\left({ }^{13} \mathrm{C}\right)$ using $\mathrm{CDCl}_{3}$ or DMSO- $d_{6}$ with tetramethylsilane as internal standard. Mass spectra were obtained by EI or FAB mode. Silica gel for chromatography was Fuji Silysia PSQ 100B. All the reactions described below were performed under argon atmosphere.

\section{5-(3-hydroxyprop-1-ynyl)-2,2-dimethyl-1,3-dioxan-5-ol (11). To a} solution of $n-\mathrm{BuMgCl}$ (39.5 mL, $35.4 \mathrm{mmol}, 0.90 \mathrm{M}$ THF solution) in THF was slowly added propargyl alcohol $(1.03 \mathrm{~mL}, 17.7 \mathrm{mmol})$ at $0{ }^{\circ} \mathrm{C}$. After the mixture was stirred at ${ }^{\circ} \mathrm{C}$ for $80 \mathrm{~min}$, the whole mixture was added to an anhydrous suspension of $\mathrm{CeCl}_{3}$ in THF $(24 \mathrm{~mL})$, which was prepared from $\mathrm{CeCl}_{3} \cdot 7 \mathrm{H}_{2} \mathrm{O}(6.70 \mathrm{~g}, 18.0 \mathrm{mmol})$ by the reported method. After stirring of $1.5 \mathrm{~h}$ at $0{ }^{\circ} \mathrm{C}$, a THF solution $(6 \mathrm{~mL})$ of 2,2,-dimethyl-1,3-dioxan-5-one $(1.53 \mathrm{~g}, 11.8 \mathrm{mmol})$ was added dropwise at $0{ }^{\circ} \mathrm{C}$ by cannula. The mixture was stirred at $0{ }^{\circ} \mathrm{C}$ for $1.5 \mathrm{~h}$. After neutralized with sat. $\mathrm{NH}_{4} \mathrm{Cl}$, the mixture was vigorously stirred for $15 \mathrm{~min}$. The resulting gummy residue was removed by decantation and filtration through celite. The filtrate was concentrated under reduced pressure and the residual solids were washed and extracted with $\mathrm{CHCl}_{3}$. The combined organic layer was dried over $\mathrm{Na}_{2} \mathrm{SO}_{4}$. After filtration, the filtrate was concentrated under reduced pressure and the residue was purified by silica gel column chromatography (33-50\% AcOEt in hexane) to give $11(1.86 \mathrm{~g}, 84 \%)$ as a white solid. ${ }^{1} \mathrm{H}-\mathrm{NMR}\left(400 \mathrm{MHz}, \mathrm{CDCl}_{3}\right) \delta 1.45(3 \mathrm{H}, \mathrm{s}), 1.48(3 \mathrm{H}, \mathrm{s}), 2.16(1 \mathrm{H}, \mathrm{t}, J=$ $6.0 \mathrm{~Hz}), 3.50(1 \mathrm{H}, \mathrm{s}), 3.77(2 \mathrm{H}, \mathrm{d}, J=11.6 \mathrm{~Hz}), 4.03(2 \mathrm{H}, \mathrm{d}, J=11.6 \mathrm{~Hz})$, $4.30(2 \mathrm{H}, \mathrm{d}, J=5.8 \mathrm{~Hz}) ;{ }^{13} \mathrm{C} \mathrm{NMR}\left(100 \mathrm{MHz}, \mathrm{CDCl}_{3}\right) \delta 18.7,27.9,50.7$, 63.2, 68.3, 82.3, 85.5, 98.6; IR (KBr) 3402.4, 1638.0, 1377.5, 1082.8, $1058.8 \mathrm{~cm}^{-1}$; EI-MS (m/z); $187\left(\mathrm{M}^{+}+1\right)$; Anal. Calcd for $\mathrm{C}_{9} \mathrm{H}_{14} \mathrm{O}_{4} \cdot 0.1 \mathrm{H}_{2} \mathrm{O}$; C, 57.50; H, 7.61. Found; C, 57.58; H, 7.69. 


\section{5-((Z)-3-tert-Butyldimethylsilyloxyprop-1-enyl)-2,2-dimethyl-1,3-dioxa}

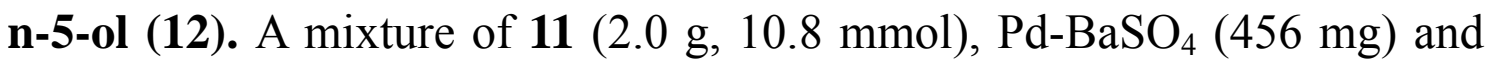
quinoline (10 drops) was stirred at room temperature for $7 \mathrm{~h}$ under $\mathrm{H}_{2}$ atmosphere. After the catalyst was removed by celite filtration, the filtrate was concentrated under reduced pressure and the residue was purified by silica gel column chromatography (60\% AcOEt in hexane) to give 12 (1.84 g, $91 \%)$ as a syrup. ${ }^{1} \mathrm{H}$ NMR $\left(400 \mathrm{MHz}, \mathrm{CDCl}_{3}\right) \delta 1.46(3 \mathrm{H}, \mathrm{s}), 1.48(3 \mathrm{H}, \mathrm{s})$, $3.37(1 \mathrm{H}, \mathrm{s}), 3.67(2 \mathrm{H}, \mathrm{d}, J=12.1 \mathrm{~Hz}), 3.90(2 \mathrm{H}, \mathrm{d}, J=11.6 \mathrm{~Hz}), 4.30(2 \mathrm{H}$, s), $5.23(1 \mathrm{H}, \mathrm{d}, J=12.6 \mathrm{~Hz}), 5.89-5.95(1 \mathrm{H}, \mathrm{m}) ;{ }^{13} \mathrm{C} \mathrm{NMR}(100 \mathrm{MHz}$, $\left.\mathrm{CDCl}_{3}\right) \delta 19.0,27.9,59.1,68.2,68.7,98.2,127.8,134.5$; IR (neat) 3390.4, 2993.7, 1651.0, 1374.9, 1199.6, $829.9 \mathrm{~cm}^{-1}$; EI-MS (m/z) $189\left(\mathrm{M}^{+}+1\right)$; HRMS Calcd for $\mathrm{C}_{14} \mathrm{H}_{19} \mathrm{~N}_{5} \mathrm{O}_{3} ; 188.1049$, Found; 188.1056 .

8,8-dimethyl-1,7,9-trioxaspiro[4.5]dec-3-ene (13). A mixture of $\mathrm{PPh}_{3}$ (399 $\mathrm{mg}, 1.52 \mathrm{mmol})$ and DEAD $(236 \mu \mathrm{L}, 1.52 \mathrm{mmol})$ in THF $(10 \mathrm{~mL})$ was stirred for $5 \mathrm{~min}$ at $0{ }^{\circ} \mathrm{C}$. To this mixture was added a solution of $\mathbf{1 2}$ (286 mg, $1.52 \mathrm{mmol})$ in THF $(2 \mathrm{~mL})$ and the mixture was stirred at $0{ }^{\circ} \mathrm{C}$ for $15 \mathrm{~min}$. After the solvents were removed under reduced pressure, the residue was purified by silica gel column chromatography $(10 \% \mathrm{AcOEt}$ in hexane). The fractions were collected and the solvents were removed under reduced pressure to leave $13(150 \mathrm{mg}, 58 \%)$ as a white crystal. $\mathrm{mp}$ 47-49 ${ }^{\circ} \mathrm{C} .{ }^{1} \mathrm{H}$ NMR $\left(400 \mathrm{MHz}, \mathrm{CDCl}_{3}\right) \delta 1.45(3 \mathrm{H}, \mathrm{s}), 1.48(3 \mathrm{H}, \mathrm{s}), 3.76(2 \mathrm{H}, \mathrm{d}, J$ $=11.6 \mathrm{~Hz}), 3.81(2 \mathrm{H}, \mathrm{d}, J=11.6 \mathrm{~Hz}), 4.71(2 \mathrm{H}, \mathrm{t}, J=1.9 \mathrm{~Hz}), 5.84-5.87$ $(1 \mathrm{H}, \mathrm{m}), 6.02(1 \mathrm{H}, \mathrm{d}, J=6.3 \mathrm{~Hz}) .{ }^{13} \mathrm{C} \mathrm{NMR}\left(100 \mathrm{MHz}, \mathrm{CDCl}_{3}\right) \delta 22.6$, 24.6, 66.7, 74.9, 98.0, 128.1, 129.1; IR (neat) 2924.2, 2853.1, 1724.1, 1215.9, 758.3 $\mathrm{cm}^{-1}$; FAB-MS (m/z); $155\left(\mathrm{M}^{+}-15\right)$. Anal. Calcd for $\mathrm{C}_{9} \mathrm{H}_{14} \mathrm{O}_{3} \cdot 0.1 \mathrm{H}_{2} \mathrm{O} ; \mathrm{C}, 62.84 ; \mathrm{H}, 8.32$. Found; $\mathrm{C}, 62.78 ; \mathrm{H}, 8.44$.

\section{5-((2R*,3R*)-3-((tert-Butyldimethylsilyloxy)methyloxiran-2-yl)-2,2-dim} ethyl-1,3-dioxan-5-ol (16). A mixture of 12 (1.59 g, $8.40 \mathrm{mmol})$, TBSCl (2.54 g, $16.8 \mathrm{mmol})$, and imidazole (1.36 g, $20.2 \mathrm{mmol})$ in $\mathrm{CH}_{2} \mathrm{Cl}_{2}(30 \mathrm{~mL})$ was stirred at room temperature for $1 \mathrm{~h}$. The mixture was diluted with 
$\mathrm{CH}_{2} \mathrm{Cl}_{2}$ and was washed with $\mathrm{H}_{2} \mathrm{O}$ and brine, then dried over $\mathrm{Na}_{2} \mathrm{SO}_{4}$. After filtration, the solvents were removed under reduced pressure to give crude 15, which was used for epoxidation without further purification. A solution of mcpba $(6.20 \mathrm{~g}, 22.2 \mathrm{mmol})$ in $\mathrm{CH}_{2} \mathrm{Cl}_{2}(50 \mathrm{~mL})$ was added to a solution of crude 15 in $\mathrm{CH}_{2} \mathrm{Cl}_{2}(30 \mathrm{~mL})$ at room temperature. The mixture was stirred at the same temperature overnight. The reaction mixture was washed with sat. $\mathrm{NaHCO}_{3}, 10 \% \mathrm{Na}_{2} \mathrm{~S}_{2} \mathrm{O}_{3}$, and brine, then dried over $\mathrm{Na}_{2} \mathrm{SO}_{4}$. After filtration, the solvents were removed under reduced pressure and the residue was purified by silica gel column chromatography $(10 \%$ AcOEt in hexane) to give 16 (2.41 g, 90\%, 2 steps) as a syrup. ${ }^{1} \mathrm{H}$ NMR (400 MHz, $\left.\mathrm{CDCl}_{3}\right) \delta 0.00(6 \mathrm{H}, \mathrm{d}, J=2.9 \mathrm{~Hz}), 0.81(9 \mathrm{H}, \mathrm{s}), 1.35(3 \mathrm{H}, \mathrm{s}), 1.39(3 \mathrm{H}, \mathrm{s})$, $3.03(1 \mathrm{H}, \mathrm{s}), 3.12(1 \mathrm{H}, \mathrm{dd}, J=9.7,4.4 \mathrm{~Hz}), 3.26(1 \mathrm{H}, \mathrm{d}, J=4.4 \mathrm{~Hz})$, 3.65-3.73 (3H, m), $3.80(1 \mathrm{H}, \mathrm{d}, J=12.1 \mathrm{~Hz}), 3.92-3.93(2 \mathrm{H}, \mathrm{m}) ;{ }^{13} \mathrm{C} \mathrm{NMR}$ $\left(100 \mathrm{MHz}, \mathrm{CDCl}_{3}\right) \delta 18.3,22.5,24.3,25.6,25.7,25.8,57.6,58.1,61.1$, 64.3, 66.7, 67.3, 98.5; IR (neat) 3444.8, 2955.9, 1373.6, 1256.2, 1079.1, $835.4 \mathrm{~cm}^{-1}$; EI-MS $(\mathrm{m} / \mathrm{z}) ; 319\left(\mathrm{M}^{+}+1\right)$; Anal. Calcd for $\mathrm{C}_{15} \mathrm{H}_{30} \mathrm{O}_{5} \mathrm{Si} ; \mathrm{C}$, 56.57; H, 9.49. Found; C, 56.39; H, 9.87.

\section{(2 $\left.R^{*}, 3 R^{*}\right)$-5-(3-(Hydroxymethyl)oxiran-2-yl)-2,2-dimethyl-1,3-dioxan-5} -ol (17). To a solution of 16 (2.37 g, $7.40 \mathrm{mmol})$ in THF $(20 \mathrm{~mL})$ was added a THF solution of TBAF $(14.8 \mathrm{~mL}, 14.8 \mathrm{mmol})$ at room temperature. The mixture was stirred at the same temperature for $1 \mathrm{~h}$. After the solvents were removed under reduced pressure, the residue was purified by silica gel column chromatography $\left(1 \% \mathrm{MeOH}\right.$ in $\left.\mathrm{CHCl}_{3}\right)$ to give $17(1.49 \mathrm{~g}$, 99\%) as a white solid. mp 69-70 ${ }^{\circ} \mathrm{C} ;{ }^{1} \mathrm{H}$ NMR $\left(400 \mathrm{MHz}, \mathrm{CDCl}_{3}\right) \delta 1.47$ $(3 \mathrm{H}, \mathrm{d}), 1.48(3 \mathrm{H}, \mathrm{s}), 2.66(1 \mathrm{H}, \mathrm{t}, J=6.5 \mathrm{~Hz}), 3.15(1 \mathrm{H}, \mathrm{d}, J=4.4 \mathrm{~Hz}), 3.28$ $(1 \mathrm{H}, \mathrm{q}, J=5.3 \mathrm{~Hz}), 3.33(1 \mathrm{H}, \mathrm{s}), 3.71-3.76(2 \mathrm{H}, \mathrm{m}), 3.86(1 \mathrm{H}, \mathrm{dd}, J=12.6$, $5.3 \mathrm{~Hz}), 3.91(1 \mathrm{H}, \mathrm{d}, J=11.6 \mathrm{~Hz}), 3.96(1 \mathrm{H}, \mathrm{d}, J=11.6 \mathrm{~Hz}), 4.06-4.12(1 \mathrm{H}$, $\mathrm{m}) ;{ }^{13} \mathrm{C} \mathrm{NMR}\left(100 \mathrm{MHz}, \mathrm{CDCl}_{3}\right) \delta 22.1,24.7,57.4,57.5,60.4,65.1,67.1$, 98.7; IR (KBr) 3449.3, 3276.2, 1372.7, 1202.51, 1074.3, 1018.7, 831.5 $\mathrm{cm}^{-1}$;EI-MS (m/z); $205\left(\mathrm{M}^{+}+1\right)$; Anal. Calcd for $\mathrm{C}_{9} \mathrm{H}_{16} \mathrm{O}_{5}$; C, 52.93; H, 7.90. Found; C, 52.84; H, 7.95 . 


\section{$\left(1 R^{*}, 5 R^{*}\right)$-2,2'-Dimethyl-3,6-dioxaspiro[bicyclo[3.1.0]hexane-2,5'-[1,3]}

dioxane] 14. A mixture of $\mathrm{PPh}_{3}(555 \mathrm{mg}, 2.22 \mathrm{mmol})$ and DEAD $(2.2 \mathrm{M}$ solution in toluene, $1.01 \mathrm{~mL}, 2.22 \mathrm{mmol}$ ) was stirred for $5 \mathrm{~min}$ at room temperature. To this mixture was added a solution of $\mathbf{1 7}(227 \mathrm{mg}, 1.11$ $\mathrm{mmol})$ in THF $(5 \mathrm{~mL})$ and the mixture was stirred at room temperature for $1.5 \mathrm{~h}$. After the solvents were removed under reduced pressure, the residue was purified by silica gel column chromatography (5\% AcOEt in hexane) to give 14 (193 mg, 94\%) as a syrup. mp 75-76 ${ }^{\circ} \mathrm{C}$; ${ }^{1} \mathrm{H}$ NMR $(400 \mathrm{MHz}$, $\left.\mathrm{CDCl}_{3}\right) \delta 1.44(3 \mathrm{H}, \mathrm{s}), 1,48(3 \mathrm{H}, \mathrm{s}), 3.68(1 \mathrm{H}, \mathrm{dd}, J=11.6,1.9 \mathrm{~Hz})$, 3.69-3.81 (4H, m), $3.91(1 \mathrm{H}, \mathrm{dd}, J=11.6,1.5 \mathrm{~Hz}), 4.01-4.04(2 \mathrm{H}, \mathrm{m}) ;{ }^{13} \mathrm{C}$ NMR $\left(100 \mathrm{MHz}, \mathrm{CDCl}_{3}\right) \delta 19.9,27.0,56.3,58.5,62.2,63.8,67.0,75.0$, 98.6; IR (KBr) 2875.7, 1374.1, 1202.5, 1079.8, $864.5 \mathrm{~cm}^{-1}$; EI-MS (m/z); $186\left(\mathrm{M}^{+}\right)$; Anal. Calcd for $\mathrm{C}_{9} \mathrm{H}_{14} \mathrm{O}_{4} ; \mathrm{C}, 58.05 ; \mathrm{H}, 7.58$. Found; C, 58.08; H, 7.65 .

(3S*, 4S*)-8,8-Dimethyl-3-(phenylthio)-1,7,9-trioxaspiro[4,5]decan-4-ol 18. To a solution of thiophenol $(2.3 \mathrm{~mL}, 21.5 \mathrm{mmol})$ in dry methanol (10 $\mathrm{mL}$ ) was added sodium methoxide $(620 \mathrm{mg}, 10.7 \mathrm{mmol})$ and the mixture was stirred at room temperature for $20 \mathrm{~min}$. To this mixture was added a solution of 14 (774 mg, $4.15 \mathrm{mmol})$ in dry methanol $(5 \mathrm{~mL})$. After being kept under reflux for $1 \mathrm{~h}$, the mixture was allowed to cool to room temperature. After the solvents were removed under reduced pressure, the residue was purified by silica gel column chromatography $(10 \%$ AcOEt in hexane) to give $18(1.09 \mathrm{~g}, 95 \%)$ as a white solid. mp 100-102 ${ }^{\circ} \mathrm{C} ;{ }^{1} \mathrm{H}$ NMR $\left(400 \mathrm{MHz}, \mathrm{CDCl}_{3}\right) \delta 1.40(3 \mathrm{H}, \mathrm{s}), 1,48(3 \mathrm{H}, \mathrm{s}), 2.75(1 \mathrm{H}, \mathrm{brs}), 3.68-3.76$ $(3 \mathrm{H}, \mathrm{m}), 3.81(1 \mathrm{H}, \mathrm{dd}, J=11.6,1.9 \mathrm{~Hz}), 3.87(1 \mathrm{H}, \mathrm{d}, J=11.6 \mathrm{~Hz}), 4.08$ $(1 \mathrm{H}, \mathrm{dd}, J=11.6,1.9 \mathrm{~Hz}), 4.25(1 \mathrm{H}, \mathrm{dd}, J=8.7,5.8 \mathrm{~Hz}), 4.32(1 \mathrm{H}, \mathrm{d}, J=$ 3.9 Hz), 7.23-7.44 (5H, m); ${ }^{13} \mathrm{C} \mathrm{NMR}\left(100 \mathrm{MHz}, \mathrm{CDCl}_{3}\right) \delta 20.3,26.7,52.9$, $62.7,66.5,70.6,78.1,80.5,98.5,127.1,129.2,130.8,134.3$; IR (KBr) 3439.2, 1200.8, 1139.0, 1078.0, 1059.0, $\mathrm{cm}^{-1}$; EI-MS (m/z); $296\left(\mathrm{M}^{+}\right)$; Anal. Calcd for $\mathrm{C}_{15} \mathrm{H}_{20} \mathrm{O}_{4} \mathrm{~S} ; \mathrm{C}, 60.79 ; \mathrm{H}, 6.80$. Found; C, 60.84; H, 6.74. 


\section{6-Chloro-9-[(3R*,4S*)-8,8-dimethyl-4-(phenylthio)-1,7,9-trioxaspiro[4.}

5]decan-3-yl]purine 20. To a solution of $18(350 \mathrm{mg}, 1.18 \mathrm{mmol}), \mathrm{PPh}_{3}$ (618 mg, $2.36 \mathrm{mmol}$ ), and 6-chloropurine (555 mg, $3.54 \mathrm{mmol})$ in THF (30 $\mathrm{mL})$ was dropwise added DEAD $(1.07 \mathrm{~mL}, 2.36 \mathrm{mmol})$ at $0{ }^{\circ} \mathrm{C}$. After the mixture was stirred at room temperature for $1 \mathrm{~h}$, the solvents were removed under reduced pressure. The residue was purified by silica gel column chromatography (60\% AcOEt in hexane) to give $20(426 \mathrm{mg}, 83 \%)$ as a white solid. mp 159-161 ${ }^{\circ} \mathrm{C} ;{ }^{1} \mathrm{H}$ NMR $\left(400 \mathrm{MHz}, \mathrm{CDCl}_{3}\right) \delta 1.44(3 \mathrm{H}, \mathrm{s})$, $1,49(3 \mathrm{H}, \mathrm{s}), 3.91(1 \mathrm{H}, \mathrm{dd}, J=12.3,1.7 \mathrm{~Hz}), 3.99(1 \mathrm{H}, \mathrm{dd}, J=12.1,1.9$ $\mathrm{Hz}), 4.08(1 \mathrm{H}, \mathrm{d}, J=12.1 \mathrm{~Hz}), 4.21(1 \mathrm{H}, \mathrm{d}, J=8.7 \mathrm{~Hz}), 4.25(1 \mathrm{H}, \mathrm{d}, J=$ $12.6 \mathrm{~Hz}), 4.36(1 \mathrm{H}, \mathrm{dd}, J=9.2,7.7 \mathrm{~Hz}), 4.41(1 \mathrm{H}, \mathrm{t}, J=8.9 \mathrm{~Hz}), 5.06(1 \mathrm{H}$, q, $J=8.4 \mathrm{~Hz}), 7.00-7.28(5 \mathrm{H}, \mathrm{m}), 8.00(1 \mathrm{H}, \mathrm{s}), 8,26(1 \mathrm{H}, \mathrm{s}) ;{ }^{13} \mathrm{C} \mathrm{NMR}(100$ $\left.\mathrm{MHz}, \mathrm{CDCl}_{3}\right) \delta 20.5,26.3,55.1,63.8,64.0,65.9,66.9,79.2,98.8,128,4$, $129.0,131.2,132.1,132.6,144.2,151.0,151.3,151.5$; uv (MeOH) $\lambda_{\max } 261$ $\mathrm{nm}$; IR (KBr) 1596.1, 1560.0, 1340.6, 1201.6, 1062.4, 828.2 cm $\mathrm{cm}^{-1}$; EI-MS $(\mathrm{m} / \mathrm{z}) ; 432\left(\mathrm{M}^{+}\right)$; Anal. Calcd for $\mathrm{C}_{20} \mathrm{H}_{21} \mathrm{ClN}_{4} \mathrm{O}_{3} \mathrm{~S} ; \mathrm{C}, 55.49 ; \mathrm{H}, 4.89 ; \mathrm{N}$, 12.94. Found; C, 55.59; H, 4.81; N, 12.87.

\section{9-[(3R*,4S*)-8,8-dimethyl-4-(phenylthio)-1,7,9-trioxaspiro[4.5]decan-3-} yl]adenine 22. A mixture of 20 (350 $\mathrm{mg}, 0.810 \mathrm{~mol})$ in $8 \mathrm{M}$ ammonia in $\mathrm{MeOH}(25 \mathrm{~mL})$ was kept at $80{ }^{\circ} \mathrm{C}$ overnight in a sealed tube. After the mixture was allowed to cool to room temperature, the solvents were removed under reduced pressure. The residue was purified by silica gel column chromatography (6\% $\mathrm{MeOH}$ in $\left.\mathrm{CHCl}_{3}\right)$ to give $22(301 \mathrm{mg}, 90 \%)$ as a white solid. mp 198-200 ${ }^{\circ} \mathrm{C} ;{ }^{1} \mathrm{H} \mathrm{NMR}\left(400 \mathrm{MHz}, \mathrm{CDCl}_{3}\right) \delta 1.39(3 \mathrm{H}$, s), 1,47 (3H, s), $3.90(1 \mathrm{H}, \mathrm{d}, J=12.6 \mathrm{~Hz}), 3.99(2 \mathrm{H}, \mathrm{s}), 4.25(1 \mathrm{H}, \mathrm{d}, J=7.3$ $\mathrm{Hz}), 4.28(1 \mathrm{H}, \mathrm{d}, J=10.6 \mathrm{~Hz}), 4.31(1 \mathrm{H}, \mathrm{dd}, J=8.7,7.3 \mathrm{~Hz}), 4.41(1 \mathrm{H}, \mathrm{t}, J$ $=9.2 \mathrm{~Hz}), 4.94(1 \mathrm{H}, \mathrm{q}, J=8.4 \mathrm{~Hz}), 5.57(2 \mathrm{H}, \mathrm{s}), 7.06-7.20(5 \mathrm{H}, \mathrm{m}), 7.67$ $(1 \mathrm{H}, \mathrm{s}), 8,25(1 \mathrm{H}, \mathrm{s}) ;{ }^{13} \mathrm{C} \mathrm{NMR}\left(100 \mathrm{MHz}, \mathrm{CDCl}_{3}\right) \delta 20.5,26.3,55.1,63.8$, 64.0, 65.9, 66.9, 79.2, 98.8, 128,4, 129.0, 131.2, 132.1, 132.6, 144.2, 151.0, 151.3, 151.5; uv (MeOH) $\lambda_{\max } 259$ nm; IR (KBr) 3150.8, 1645.2, 1601.4, 
$1091.3 \mathrm{~cm}^{-1}$; EI-MS $(\mathrm{m} / \mathrm{z}) ; 413\left(\mathrm{M}^{+}\right)$; Anal. Calcd for $\mathrm{C}_{20} \mathrm{H}_{23} \mathrm{~N}_{5} \mathrm{O}_{3} \mathrm{~S}$; C, 58.09; H, 5.61; N, 16.94. Found; C, 58.28; H, 5.57; N, 16.61.

(S*)-9-[8,8-dimethyl-1,7,9-trioxaspiro[4.5]decan-3-yl]adenine 23. To a solution of $22(250 \mathrm{mg}, 0.610 \mathrm{mmol})$ in toluene $(10 \mathrm{~mL})$ were added $\mathrm{Bu}_{3} \mathrm{SnH}(320 \mu \mathrm{L}, 1.22 \mathrm{mmol})$ and AIBN (103 mg, $\left.0.610 \mathrm{mmol}\right)$. The mixture was kept under reflux for $10 \mathrm{~h}$. After the solvents were removed under reduced pressure, the residue was purified by silica gel column chromatography $\left(1 \% \mathrm{MeOH}\right.$ in $\left.\mathrm{CHCl}_{3}\right)$ to give $23(162 \mathrm{mg}, 87 \%)$ as a white solid. mp 252-254 ${ }^{\circ} \mathrm{C} ;{ }^{1} \mathrm{H}$ NMR (400 MHz, DMSO-d $\left.d_{6}\right) \delta 1.31(3 \mathrm{H}, \mathrm{s})$, $1.33(3 \mathrm{H}, \mathrm{s}), 2.17(1 \mathrm{H}, \mathrm{dd}, J=13.5,6.3 \mathrm{~Hz}), 2.37(1 \mathrm{H}, \mathrm{dd}, J=13.5,7.7 \mathrm{~Hz})$, 3.66-3.78 (4H, m), $4.16(2 \mathrm{H}, \mathrm{m}), 5.06-5.12(1 \mathrm{H}, \mathrm{m}), 7.24(1 \mathrm{H}, \mathrm{s}), 8,13(1 \mathrm{H}$, s), $8.18(1 \mathrm{H}, \mathrm{s}) ;{ }^{13} \mathrm{C}$ NMR (100 MHz, DMSO-d $\left.d_{6}\right) \delta 22.3,24.8,37.5,53.5$, 65.6, 66.3, 69.4, 77.0, 97.3, 119.0, 139.0, 149.9, 152.4, 156.0; uv (MeOH) $\lambda_{\max } 261 \mathrm{~nm}$; IR (KBr) 3388.7, 1664.2, 1599.5, $1081.4 \mathrm{~cm}^{-1}$; EI-MS (m/z); $305\left(\mathrm{M}^{+}\right)$; HRMS Calcd for $\mathrm{C}_{14} \mathrm{H}_{19} \mathrm{~N}_{5} \mathrm{O}_{3} ; 305.1488$, Found; 305.1484 .

\section{(S*)-4-[(Adenin-9-yl)-tetrahydrofuran-2,2-diyl]dimethanol}

(( \pm )-2',3'-Dideoxy-4'-hydroxymethyl-iso-adenosine, 24) A mixture of 23 (50 $\mathrm{mg}, 0.16 \mathrm{mmol}$ ) in $80 \%$ aq. AcOH was stirred at room temperature for $5 \mathrm{~h}$. After the solvents were removed under reduced pressure, the residual solvents were further removed by repeating co-evaporation with EtOH 5 times. The residue was purified by silica gel column chromatography $(5 \%$ $\mathrm{MeOH}$ in $\mathrm{CHCl}_{3}$ ) to give 24 (39 mg, 94\%) as a white solid. mp 230-232 ${ }^{\circ} \mathrm{C} ;{ }^{1} \mathrm{H}$ NMR (400 MHz, DMSO-d 6 ) $\delta 2.22(1 \mathrm{H}, \mathrm{dd}, J=13.0,6.8 \mathrm{~Hz}), 2.41$ $(1 \mathrm{H}, \mathrm{dd}, J=13.0,8.2 \mathrm{~Hz}), 3.37-3.45(4 \mathrm{H}, \mathrm{m}), 4.05(1 \mathrm{H}, \mathrm{dd}, J=9.2,6.3$ $\mathrm{Hz}), 4.21(1 \mathrm{H}, J=8.7,6.3 \mathrm{~Hz}), 4.82-4.86(2 \mathrm{H}, \mathrm{m}), 5.09-5.16(1 \mathrm{H}, \mathrm{m}), 7.22$ $(2 \mathrm{H}, \mathrm{s}), 8.12(1 \mathrm{H}, \mathrm{s}), 8.26(1 \mathrm{H}, \mathrm{s}) ;{ }^{13} \mathrm{C}$ NMR $\left(100 \mathrm{MHz}, \mathrm{DMSO}-d_{6}\right) \delta 35.2$, $54.2,63.8,63.9,86.4,118.8,139.0,149.4,152.3,156.0$; uv $(\mathrm{MeOH}) \lambda_{\max }$ $261 \mathrm{~nm}$; IR (KBr) 3369.5, 3195.1, 1656.2, 1612.1, 1312.9, $1050.7 \mathrm{~cm}^{-1}$; FAB-MS $(\mathrm{m} / \mathrm{z}) ; 266\left(\mathrm{M}^{+}+1\right)$; HRMS Calcd for $\mathrm{C}_{11} \mathrm{H}_{15} \mathrm{~N}_{5} \mathrm{O}_{3}$ : 266.1253, Found: 266.1252 


\section{3-Benzoyl-1-[(3R*,4S*)-8,8-dimethyl-4-(phenylthio)-1,7,9-trioxaspiro[4}

.5]decan-3-yl]thymine 21 . To a solution of 18 (250 mg, $0.840 \mathrm{mmol}), \mathrm{PPh}_{3}$ (240 mg, $0.920 \mathrm{mmol})$, and $N^{3}$-benzoylthymine (288 $\left.\mathrm{mg}, 1.26 \mathrm{mmol}\right)$ in THF $(20 \mathrm{~mL})$ was dropwise added DEAD $(417 \mu \mathrm{L}, 1.26 \mathrm{mmol})$ at $0{ }^{\circ} \mathrm{C}$. After the mixture was stirred at room temperature for $3 \mathrm{~h}$, the solvents were removed under reduced pressure. The residue was purified by silica gel column chromatography (60\% AcOEt in hexane) to give 21 (223 mg, 52\%) as a white solid. mp $94-96{ }^{\circ} \mathrm{C} ;{ }^{1} \mathrm{H}$ NMR $\left(400 \mathrm{MHz}, \mathrm{CDCl}_{3}\right) \delta 1.38(1 \mathrm{H}, \mathrm{s})$, $1.44(1 \mathrm{H}, \mathrm{s}), 1.82(1 \mathrm{H}, \mathrm{s}), 3.71(1 \mathrm{H}, \mathrm{d}, J=8.7 \mathrm{~Hz}), 3.76-3.84(2 \mathrm{H}, \mathrm{m}), 3.96$ $(1 \mathrm{H}, \mathrm{d}, J=12.6 \mathrm{~Hz}), 4.04(1 \mathrm{H}, \mathrm{dd}, J=9.7,7.2 \mathrm{~Hz}), 4.17-4.22(2 \mathrm{H}, \mathrm{m})$, $4.88(1 \mathrm{H}, \mathrm{q}, J=7.7 \mathrm{~Hz}), 6.89(1 \mathrm{H}, \mathrm{s}), 7.30-7.37(3 \mathrm{H}, \mathrm{m}), 7.46-7.50(4 \mathrm{H}, \mathrm{m})$, $7.63(1 \mathrm{H}, \mathrm{m}), 7.82-7.85(2 \mathrm{H}, \mathrm{m}) ;{ }^{13} \mathrm{C} \mathrm{NMR}\left(100 \mathrm{MHz}, \mathrm{CDCl}_{3}\right) \delta 12.5,20.0$, 26.8, 63.2, 65.8, 66.2, 79.4, 98.7, 111.8, 128.6, 129.1, 129.7, 130.4, 131.4, $132.3,132.9,135.1,137.1,149.4,162.2,168.4$; uv (MeOH) $\lambda_{\max } 254 \mathrm{~nm}$; IR (KBr) 1749.0, 1696.3, $1657.6 \mathrm{~cm}^{-1}$; EI-MS (m/z): $508\left(\mathrm{M}^{+}\right)$; HRMS Calcd for $\mathrm{C}_{27} \mathrm{H}_{28} \mathrm{~N}_{2} \mathrm{O}_{6} \mathrm{~S} ; 508.1668$, Found; 508.1664

\section{1-[(3R*,4S*)-8,8-Dimethyl-4-(phenylthio)-1,7,9-trioxaspiro[4.5]decan-3} -yl]thymine 25. A mixture of 21 (214 $\mathrm{mg}, 0.420 \mathrm{~mol})$ in conc. aq. ammonia $(10 \mathrm{~mL})$ and $\mathrm{MeOH}(10 \mathrm{~mL})$ was stirred at room temperature for $2 \mathrm{~h}$. After the solvents were removed under reduced pressure, the residue was purified by silica gel column chromatography (50\% AcOEt in hexane) to give 25 (160 mg, 95\%) as a white solid. mp 165-167 ${ }^{\circ} \mathrm{C} ;{ }^{1} \mathrm{H}$ NMR (400 $\left.\mathrm{MHz}, \mathrm{CDCl}_{3}\right) \delta 1.39(1 \mathrm{H}, \mathrm{s}), 1.45(1 \mathrm{H}, \mathrm{s}), 1.80(1 \mathrm{H}, \mathrm{s}), 3.68(1 \mathrm{H}, \mathrm{d}, J=8.7$ $\mathrm{Hz}), 3.81-3.87(2 \mathrm{H}, \mathrm{m}), 3.95-4.01(2 \mathrm{H}, \mathrm{m}), 4.16-4.23(2 \mathrm{H}, \mathrm{m}), 4.95(1 \mathrm{H}, \mathrm{q}$, $J=7.9 \mathrm{~Hz}), 6.83(1 \mathrm{H}, \mathrm{s}), 7.26-7.29(3 \mathrm{H}, \mathrm{m}), 7.44-7.46(2 \mathrm{H}, \mathrm{m}), 9.63(1 \mathrm{H}$, s); ${ }^{13} \mathrm{C}$ NMR (100 MHz, $\left.\mathrm{CDCl}_{3}\right) \delta 12.3,19.9,26.7,54.8,63.0,64.7,65.8$, 66.3, 79.4, 98.6, 111.7, 128.4, 129.3, 132.2, 132.9, 137.0, 150.5, 163.5; uv $(\mathrm{MeOH}) \lambda_{\max } 261 \mathrm{~nm}$; IR (KBr) 3455.6, 1695.1, 1090.5, $1056.0 \mathrm{~cm}^{-1}$; EI-MS (m/z); $404\left(\mathrm{M}^{+}\right)$; HRMS Calcd for $\mathrm{C}_{20} \mathrm{H}_{24} \mathrm{~N}_{2} \mathrm{O}_{5} \mathrm{~S}$; 404.1406, Found; 404.1410 
(S*)-1-[8,8-dimethyl-1,7,9-trioxaspiro[4.5]decan-3-yl]thymine 26. To a solution of $25(154 \mathrm{mg}, 0.380 \mathrm{mmol})$ and $\mathrm{Bu}_{3} \mathrm{SnH}(298 \mu \mathrm{L}, 1.14 \mathrm{mmol})$ in toluene $(10 \mathrm{~mL})$ was added a solution of AIBN (64 mg, $0.380 \mathrm{mmol})$ in toluene $(5 \mathrm{~mL})$ over $1 \mathrm{~h}$. The mixture was kept under reflux for $2 \mathrm{~h}$. After the solvents were removed under reduced pressure, the residue was purified by silica gel column chromatography (70\% AcOEt in hexane) to give 26 (82 mg, 74\%) as a white solid. mp 252-253 ${ }^{\circ} \mathrm{C} ;{ }^{1} \mathrm{H}$ NMR (400 MHz, DMSO-d $)_{6} \delta 1.29(3 \mathrm{H}, \mathrm{s}), 1.32(3 \mathrm{H}, \mathrm{s}), 1.73(1 \mathrm{H}, \mathrm{dd}, J=13.5,5.8 \mathrm{~Hz})$, $1.78(1 \mathrm{H}, \mathrm{s}), 2.16(1 \mathrm{H}, \mathrm{dd}, J=14.0,8.7 \mathrm{~Hz}), 3.62(2 \mathrm{H}, \mathrm{s}), 3.69(1 \mathrm{H}, \mathrm{d}, J=$ $12.1 \mathrm{~Hz}), 3.79(1 \mathrm{H}, \mathrm{d}, J=12.1 \mathrm{~Hz}), 3.87(1 \mathrm{H}, \mathrm{dd}, J=10.1,5.3 \mathrm{~Hz}), 3.96$ $(1 \mathrm{H}, \mathrm{dd}, J=9.7,6.8 \mathrm{~Hz}), 4.91-4.98(1 \mathrm{H}, \mathrm{m}), 7.47(1 \mathrm{H}, \mathrm{s}), 11.26(1 \mathrm{H}, \mathrm{s})$; ${ }^{13} \mathrm{C}$ NMR (100 MHz, DMSO-d $\left.d_{6}\right) \delta 12.2,22.0,25.1,37.0,54.2,65.1,66.3$, 68.4, 77.0, 97.2, 109.5, 137.3, 150.9, 163.7; uv (MeOH) $\lambda_{\max } 271 \mathrm{~nm}$; IR (KBr) 3175.8, 1689.5, 1672.4, 1282.5, 1089.9, $1050.6 \mathrm{~cm}^{-1}$; EI-MS (m/z); $296\left(\mathrm{M}^{+}\right)$; HRMS Calcd for $\mathrm{C}_{14} \mathrm{H}_{20} \mathrm{~N}_{2} \mathrm{O}_{5} ; 296.1372$, Found; 296.1378

\section{(S*)-4-[(Thymin-1-yl)-tetrahydrofuran-2,2-diyl]dimethanol}

(( \pm )-3’-Deoxy-4'-hydroxymethyl-iso-thymidine, 27) A mixture of 26 (47 $\mathrm{mg}, 0.16 \mathrm{mmol}$ ) in $80 \%$ aq. AcOH was stirred at room temperature for $6 \mathrm{~h}$. After the solvents were removed under reduced pressure, the residual solvents were further removed by repeating co-evaporation with EtOH 5 times. The residue was purified by silica gel column chromatography $(5 \%$ $\mathrm{MeOH}$ in $\mathrm{CHCl}_{3}$ ) to give 27 (40 mg, 98\%) as a white solid. mp 195-198 ${ }^{\circ} \mathrm{C} ;{ }^{1} \mathrm{H}$ NMR (400 MHz, DMSO- $\left.d_{6}\right) \delta 1.76(3 \mathrm{H}, \mathrm{s}), 1.87(1 \mathrm{H}, \mathrm{dd}, J=13.5$, $6.3 \mathrm{~Hz}), 2.20(1 \mathrm{H}, \mathrm{dd}, J=13.5,9.2 \mathrm{~Hz}), 3.24-3.30(2 \mathrm{H}, \mathrm{m}), 3.38-3.50(2 \mathrm{H}$, m), $3.78(1 \mathrm{H}, \mathrm{dd}, J=9.4,5.1 \mathrm{~Hz}), 4.00(1 \mathrm{H}, \mathrm{dd}, J=9.7,7.3 \mathrm{~Hz}), 4.76(1 \mathrm{H}$, $\mathrm{t}, J=5.8 \mathrm{~Hz}), 4.89(1 \mathrm{H}, \mathrm{t}, J=5.8 \mathrm{~Hz}), 5.04-5.11(1 \mathrm{H}, \mathrm{m}), 7.64(1 \mathrm{H}, \mathrm{s})$, $11.22(1 \mathrm{H}, \mathrm{s}) ;{ }^{13} \mathrm{C}$ NMR (100 MHz, DMSO-d 6 ) $\delta 12.2,34.3,54.6,63.4$, 70.1, 86.6, 109.1, 137.7, 150.9, 163.7; uv $(\mathrm{MeOH}) \lambda_{\max } 271 \mathrm{~nm}$; IR $(\mathrm{KBr})$ 3390.0, 1681.6, 1473.6, 1062.1, 1040.2 $\mathrm{cm}^{-1}$; FAB-MS (m/z); $257\left(\mathrm{M}^{+}+1\right)$; HRMS Calcd for $\mathrm{C}_{11} \mathrm{H}_{16} \mathrm{~N}_{2} \mathrm{O}_{5} ; 257.1131$, Found; 257.1137. 


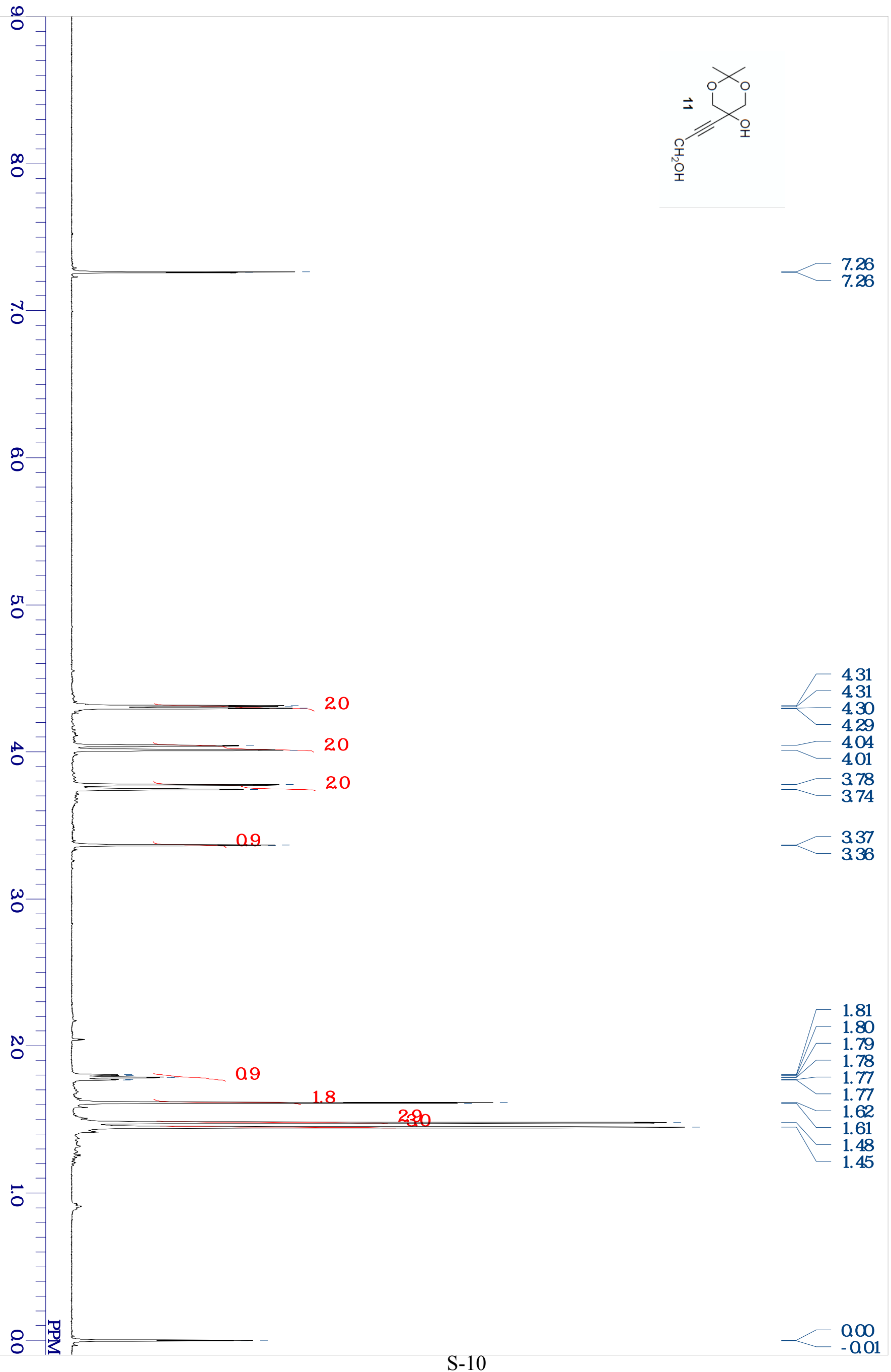




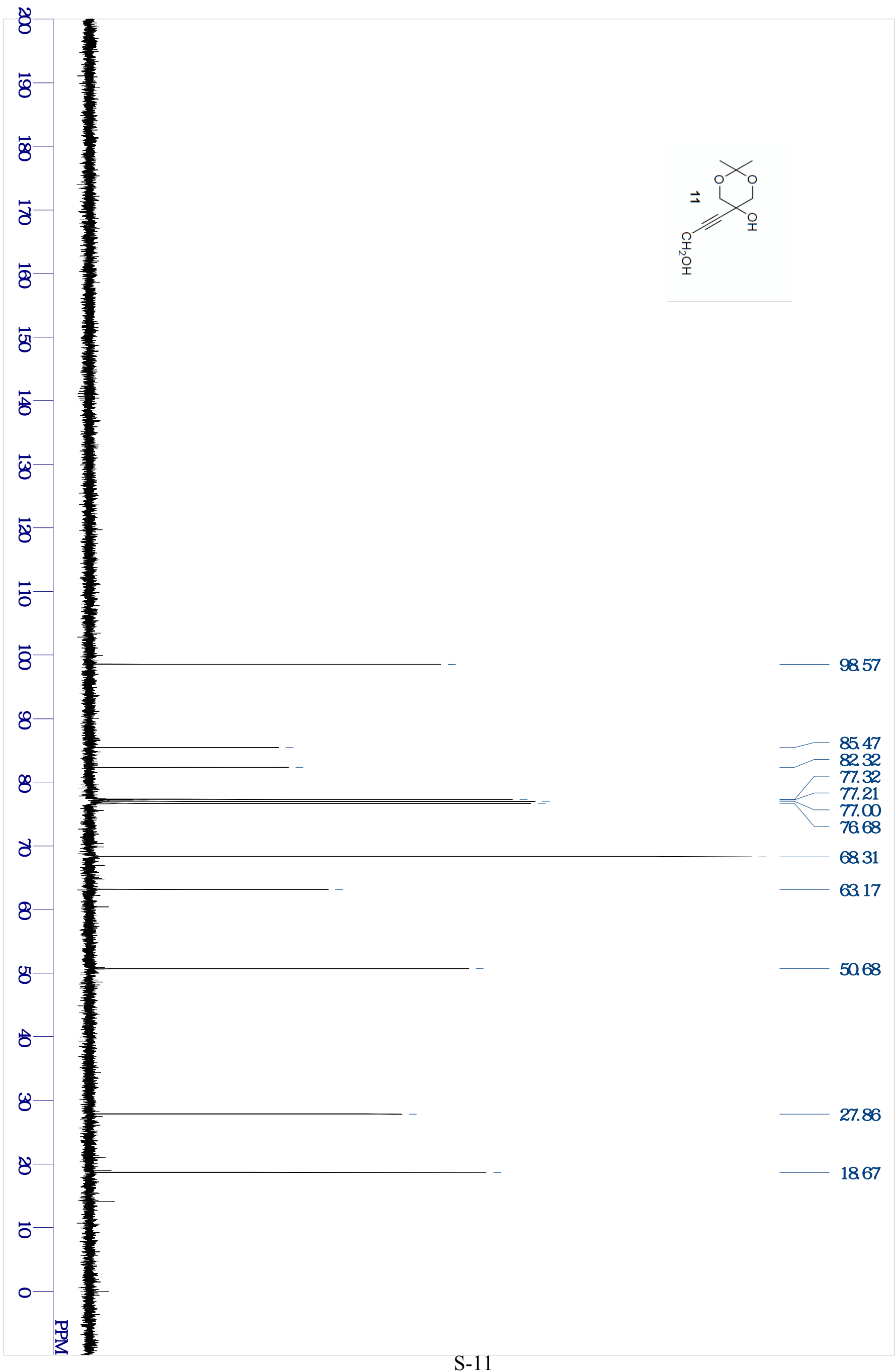




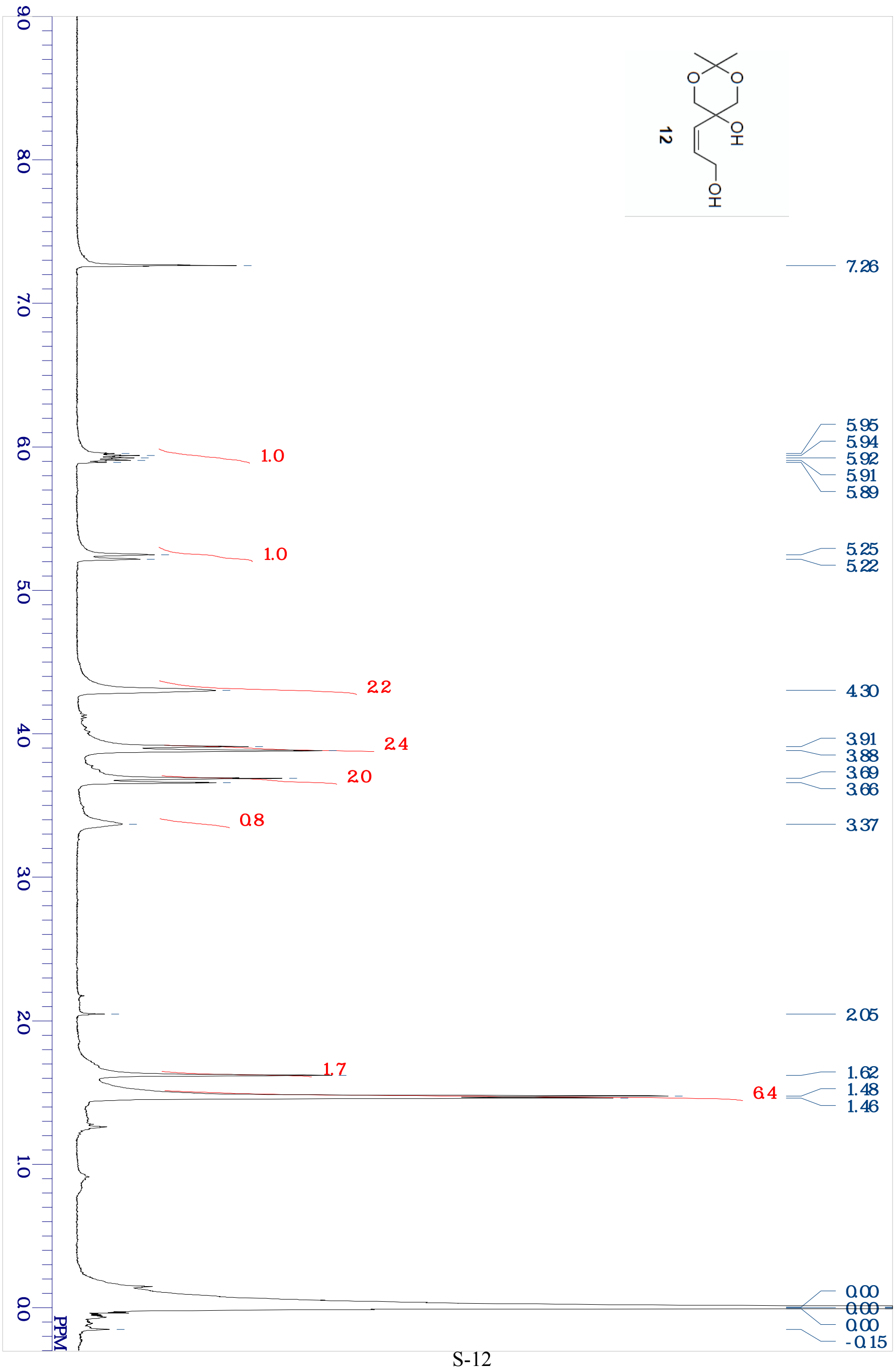




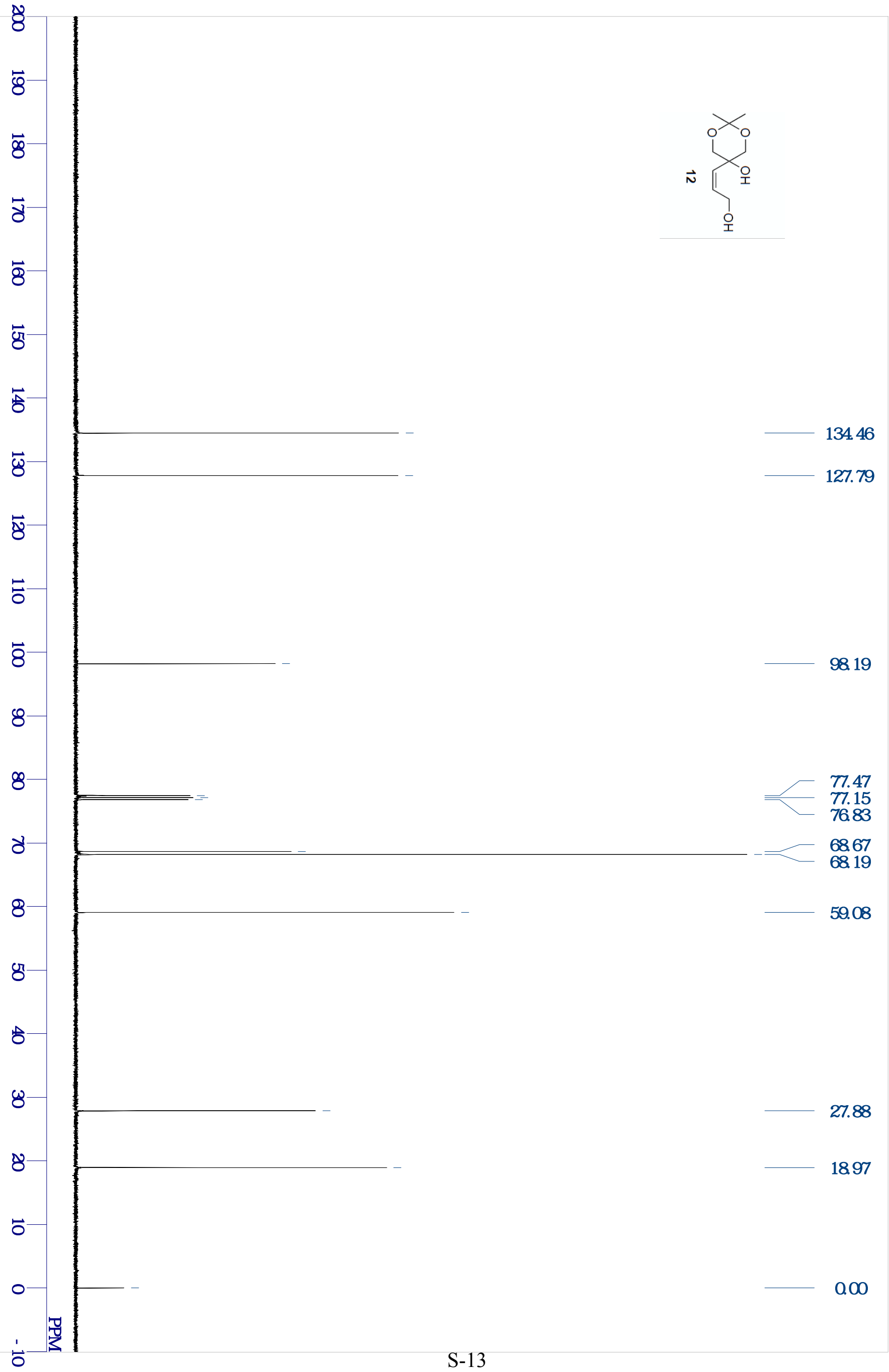




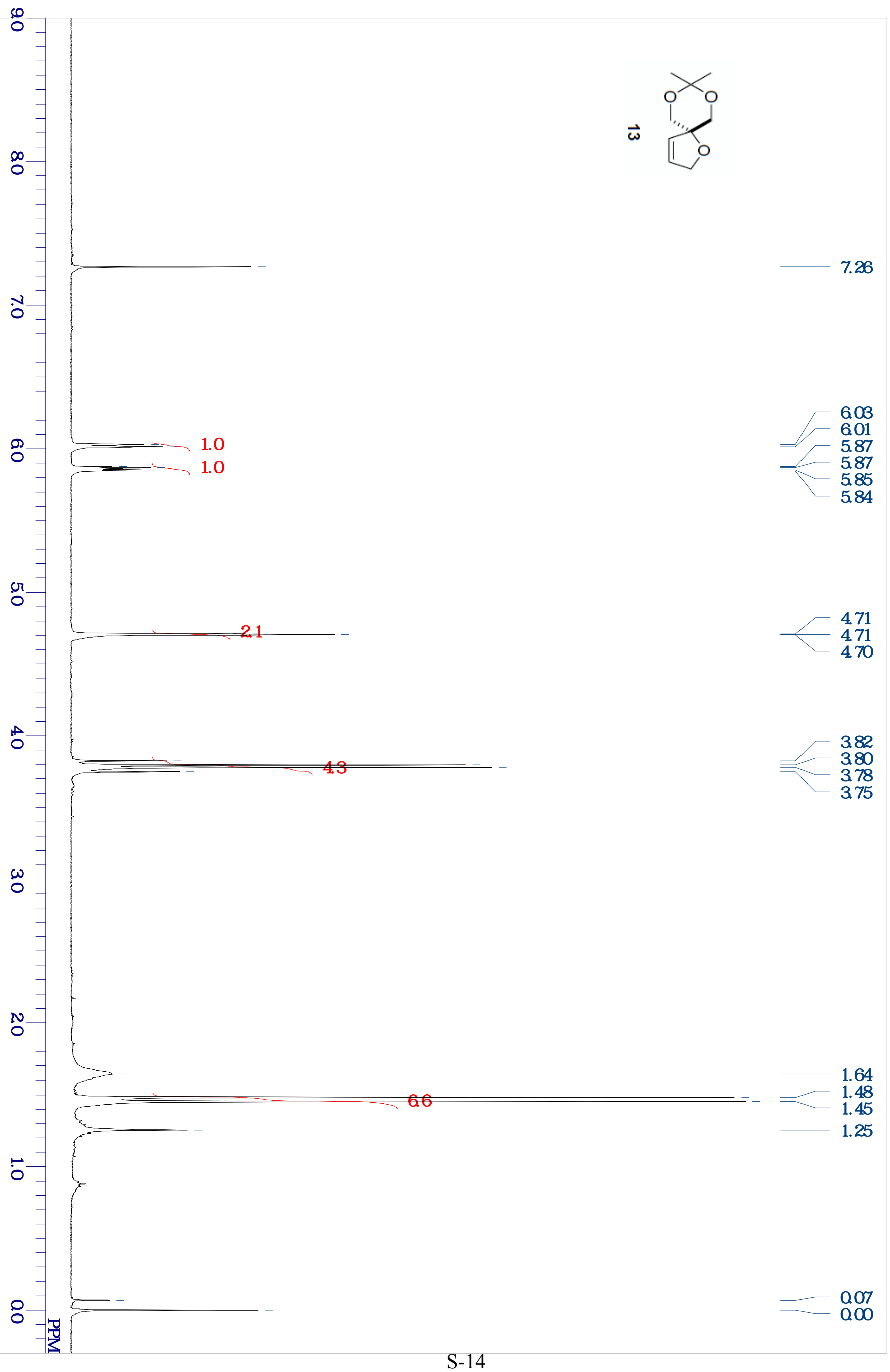




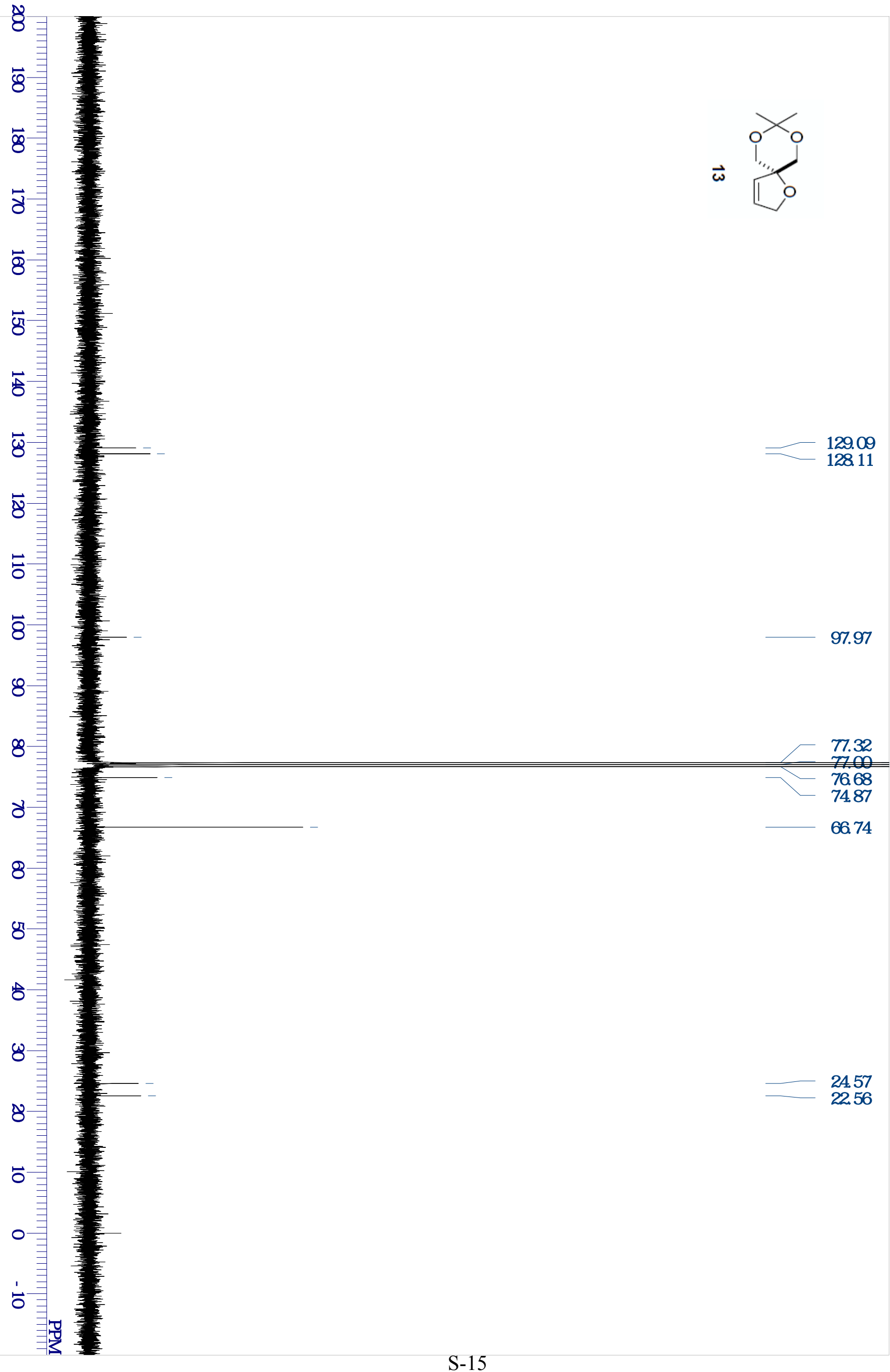




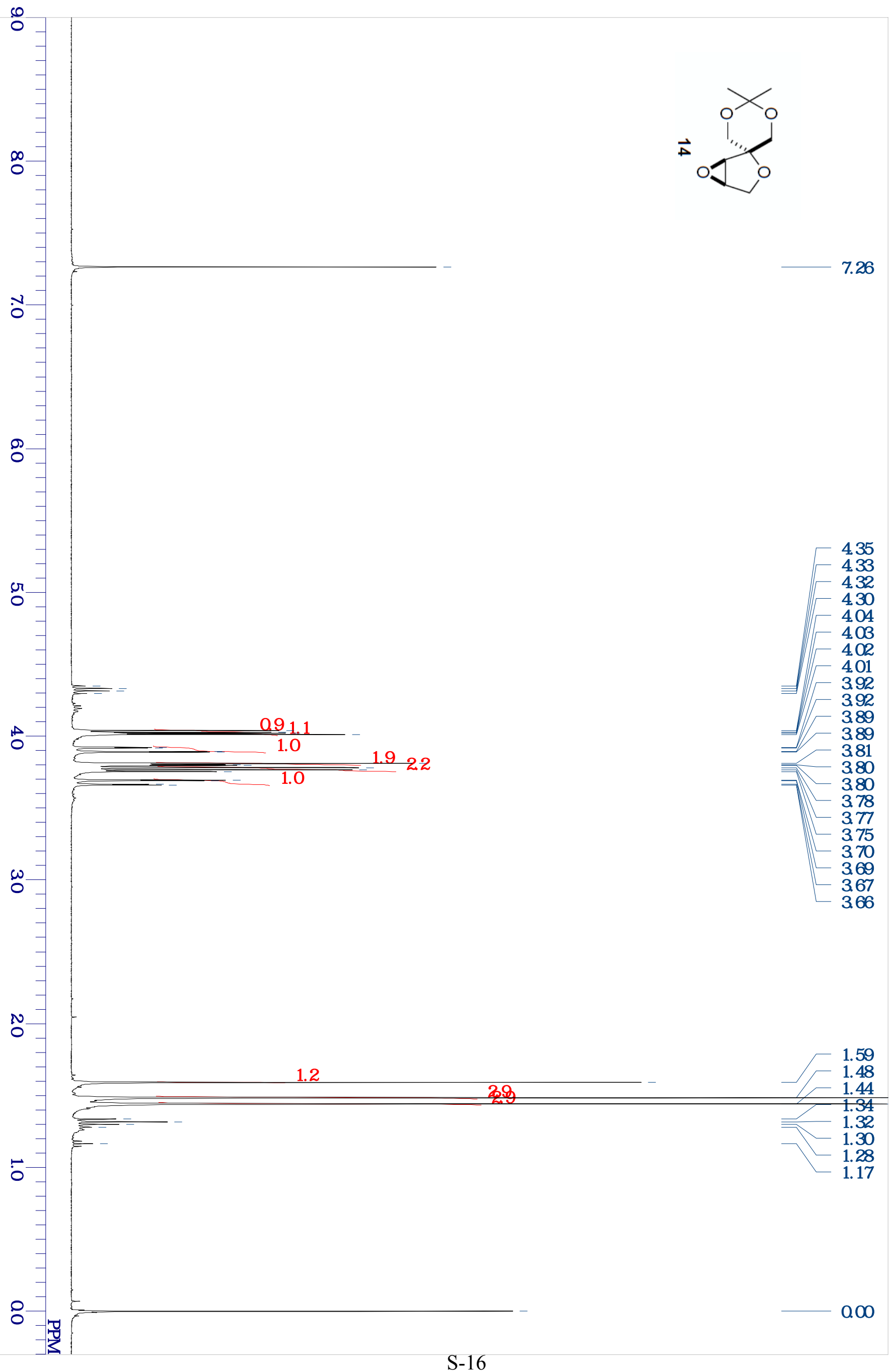




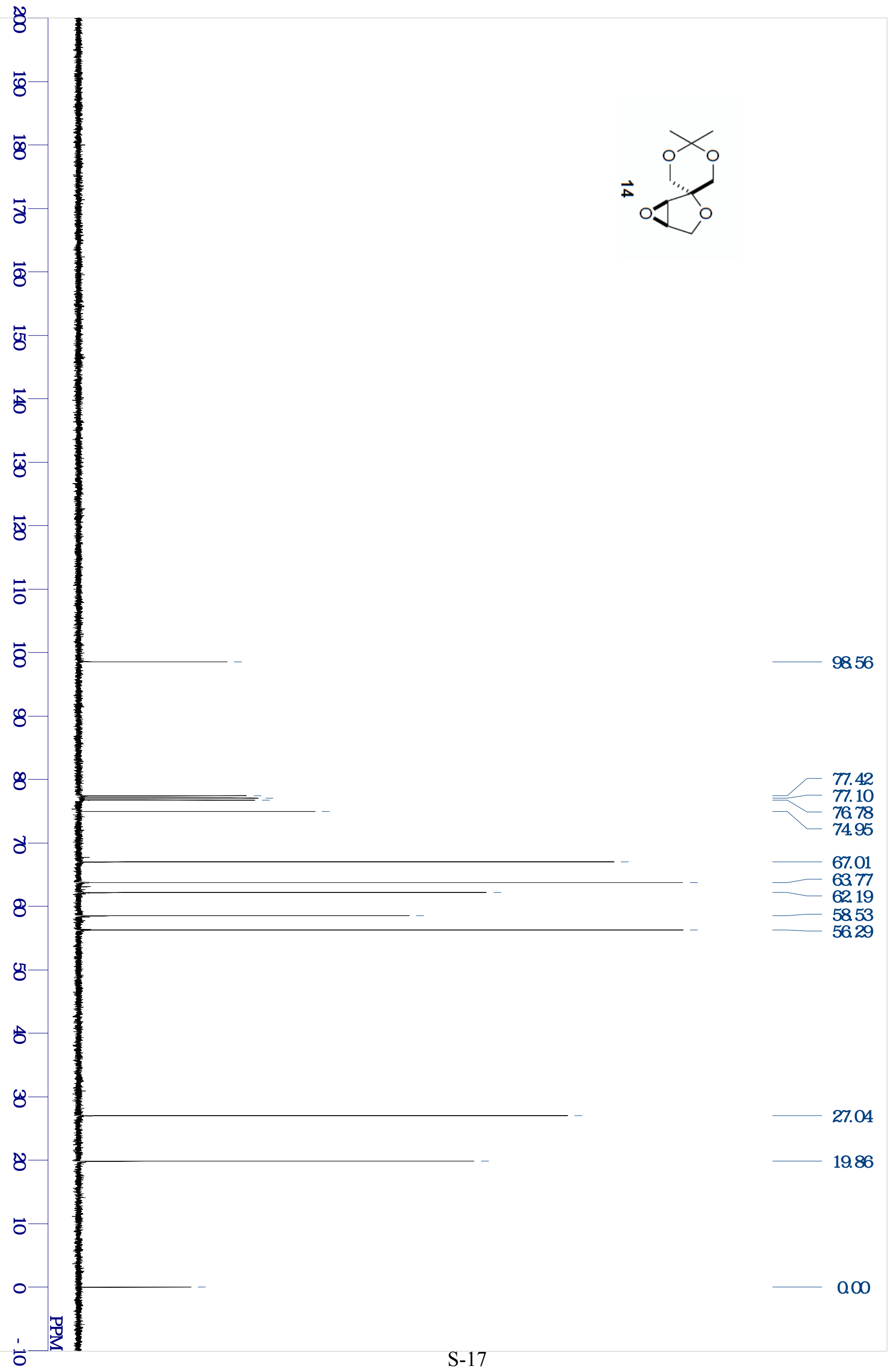




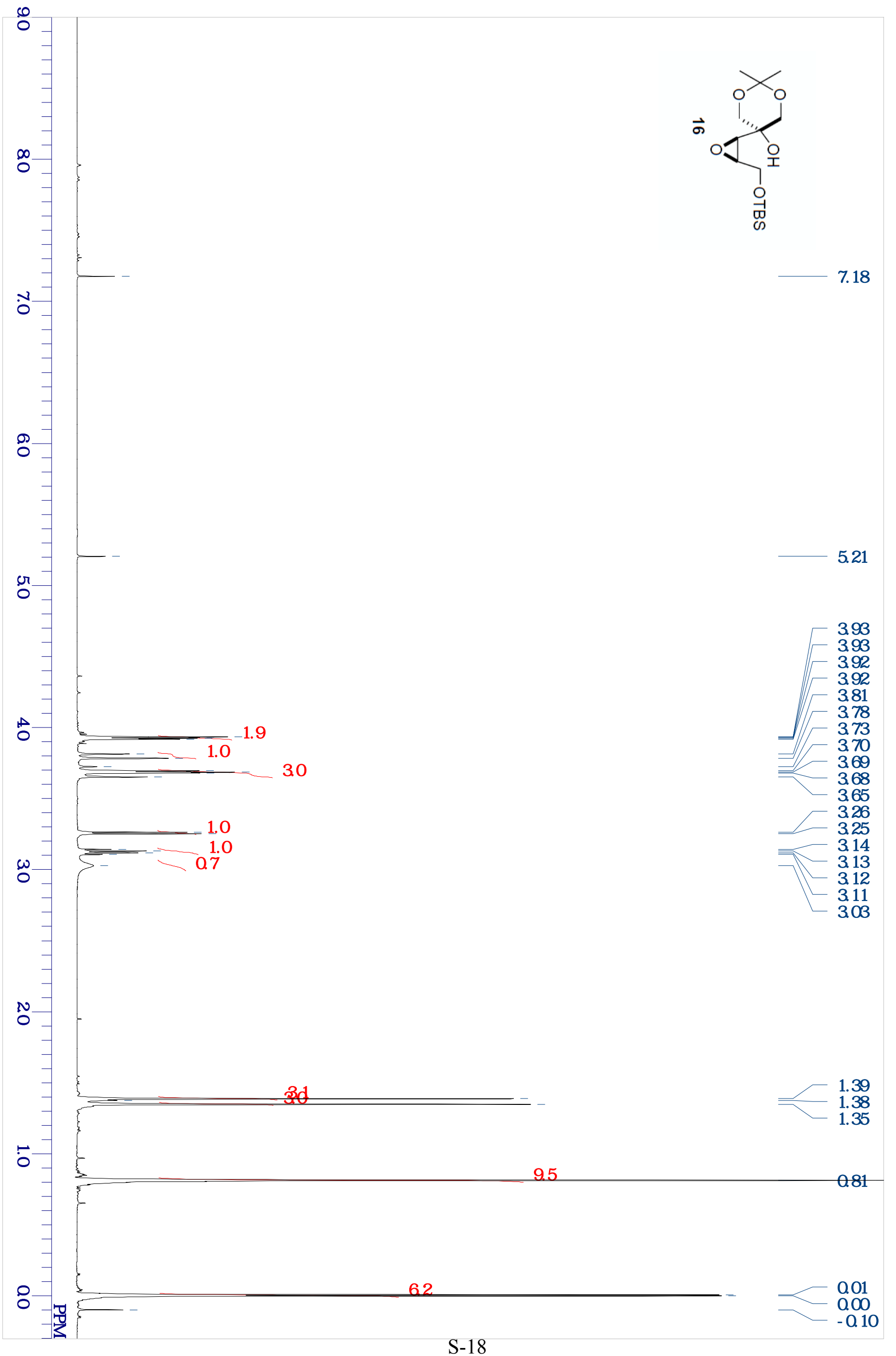




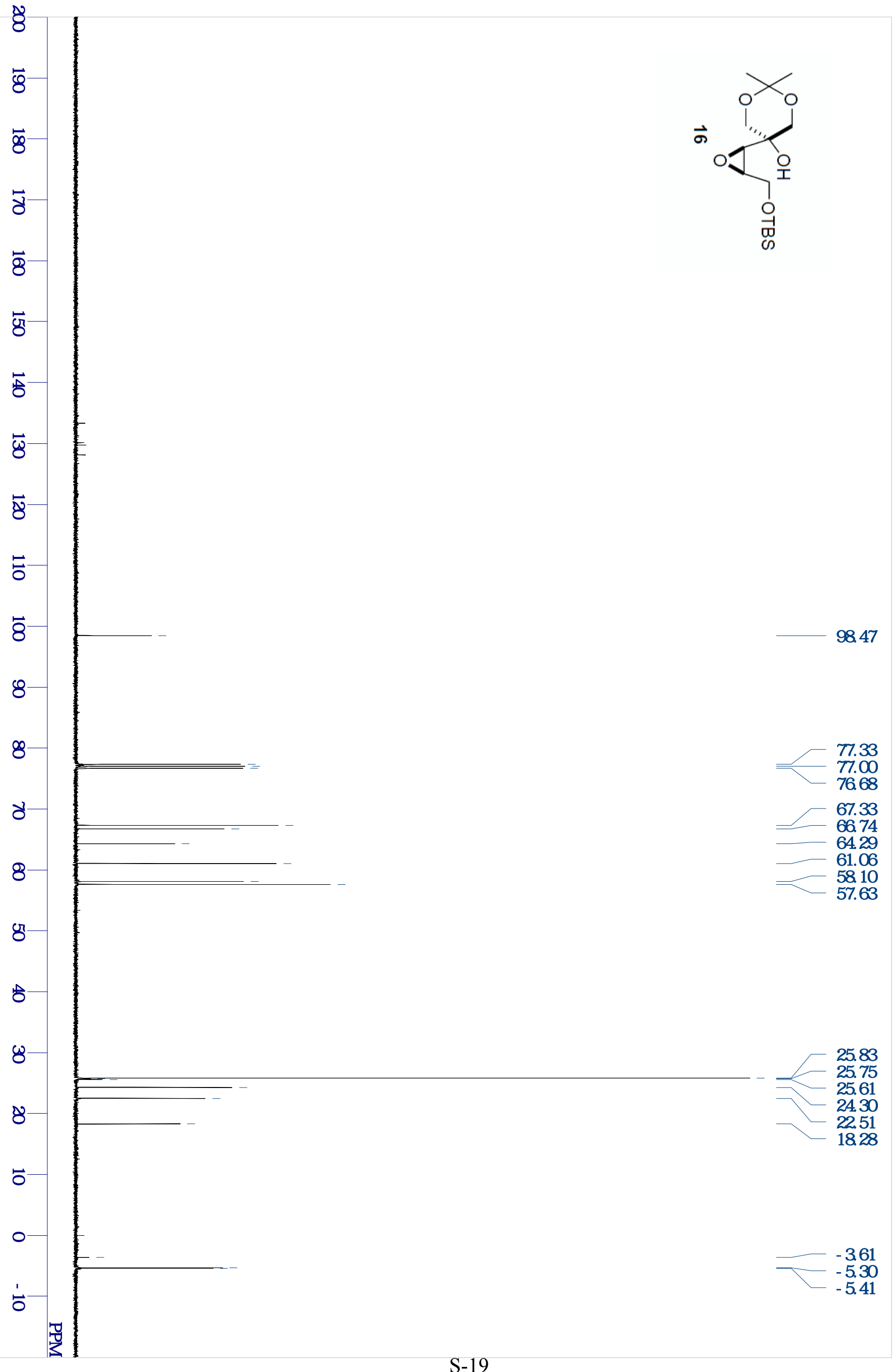




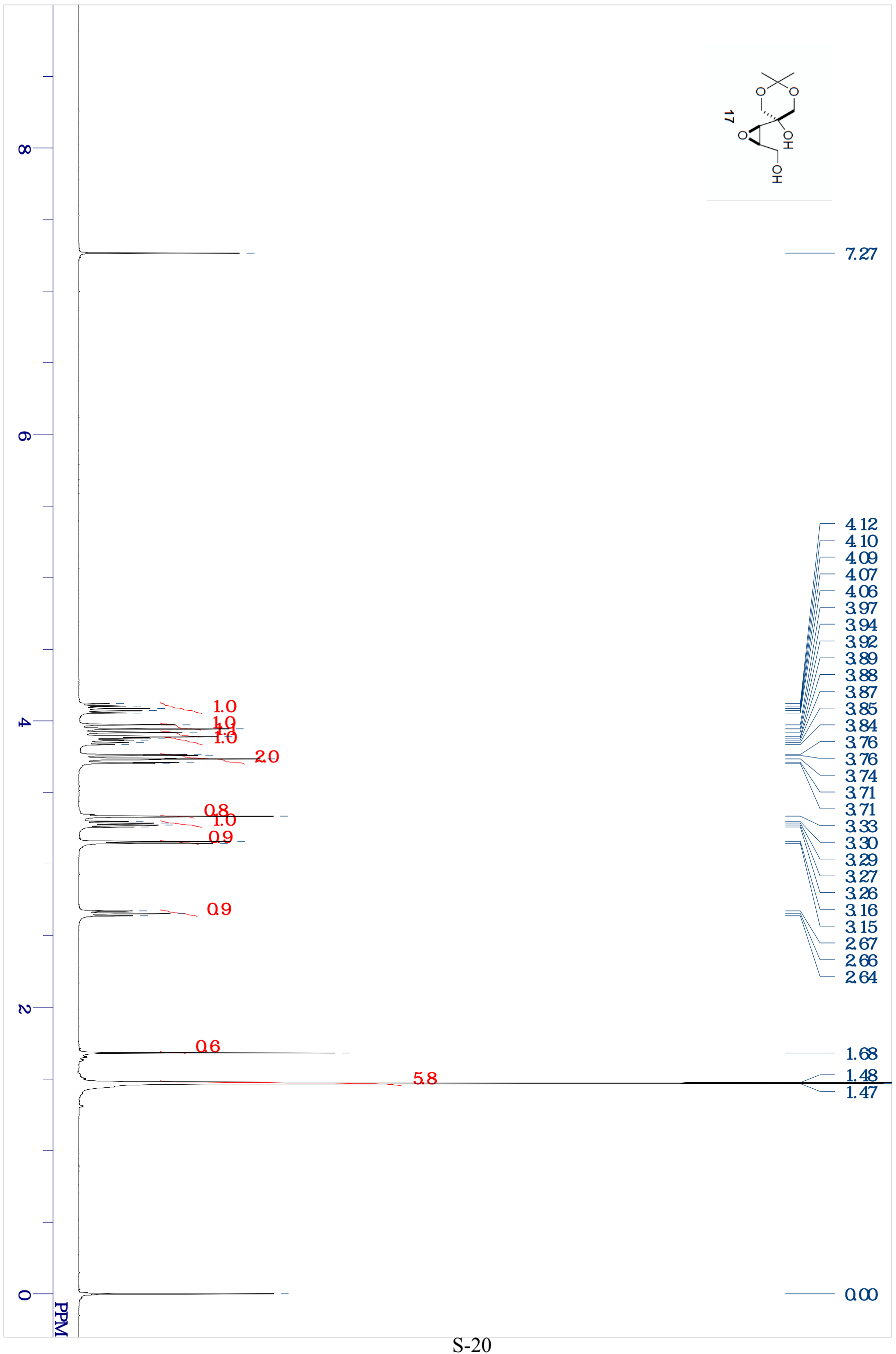




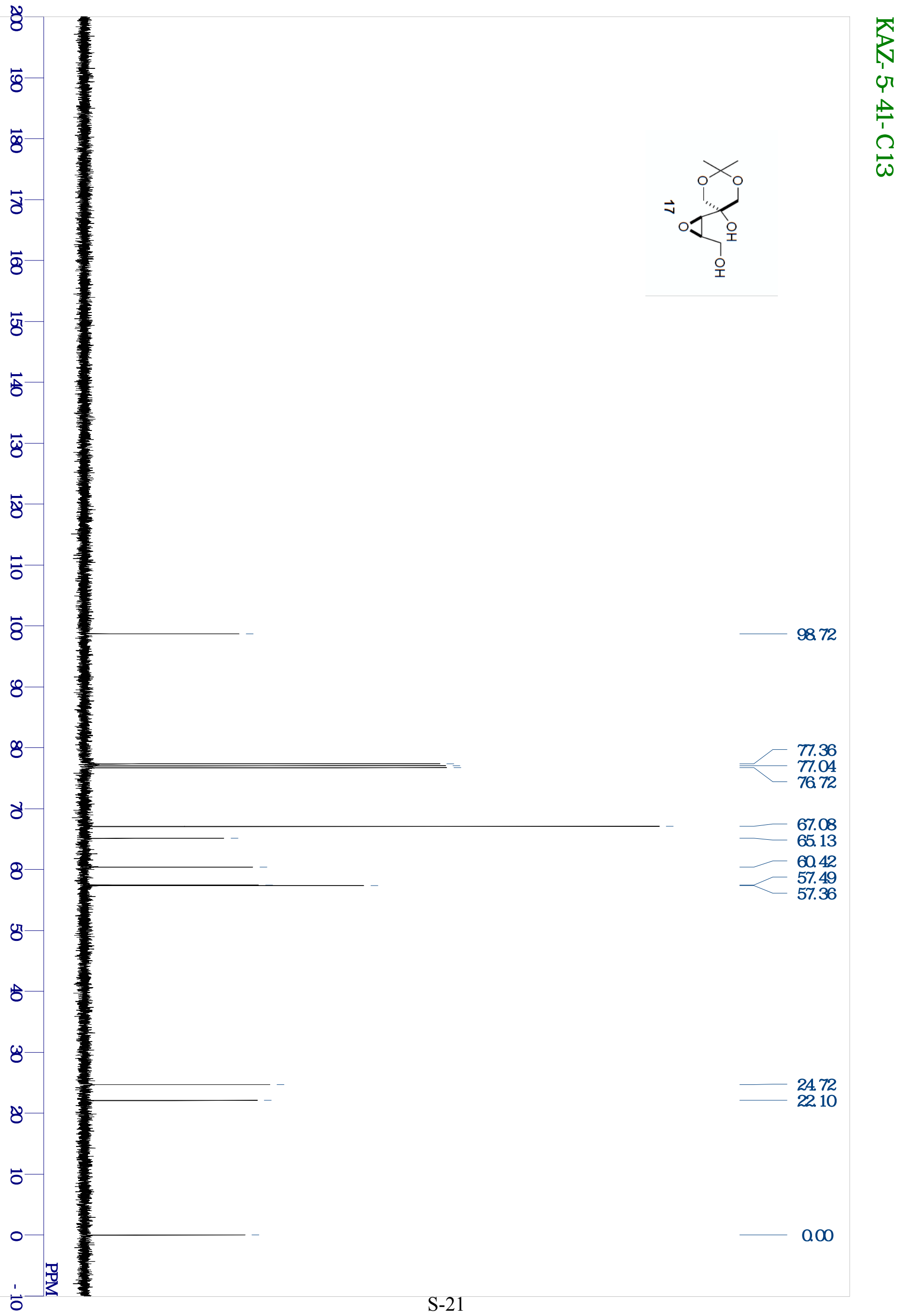




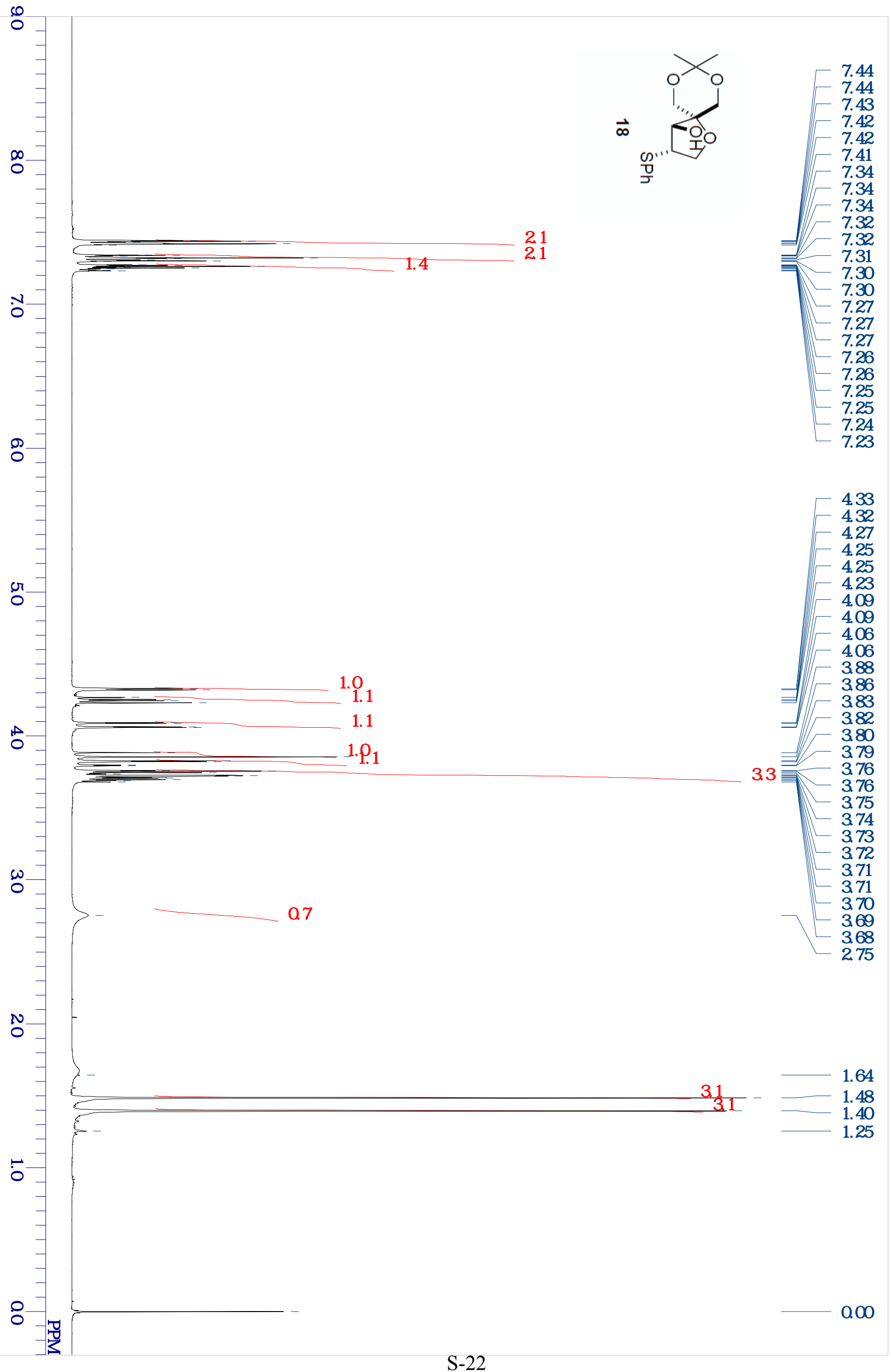




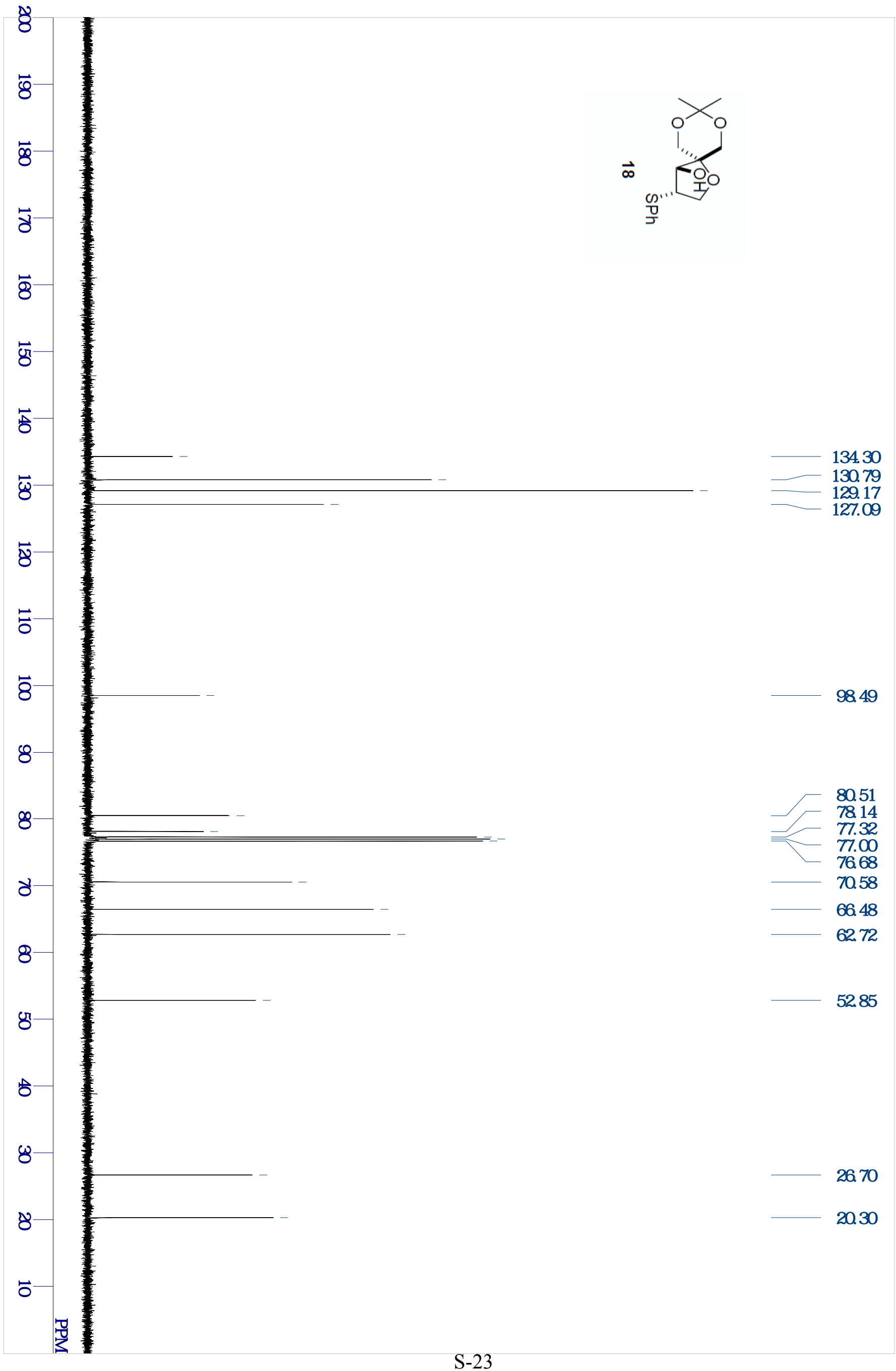




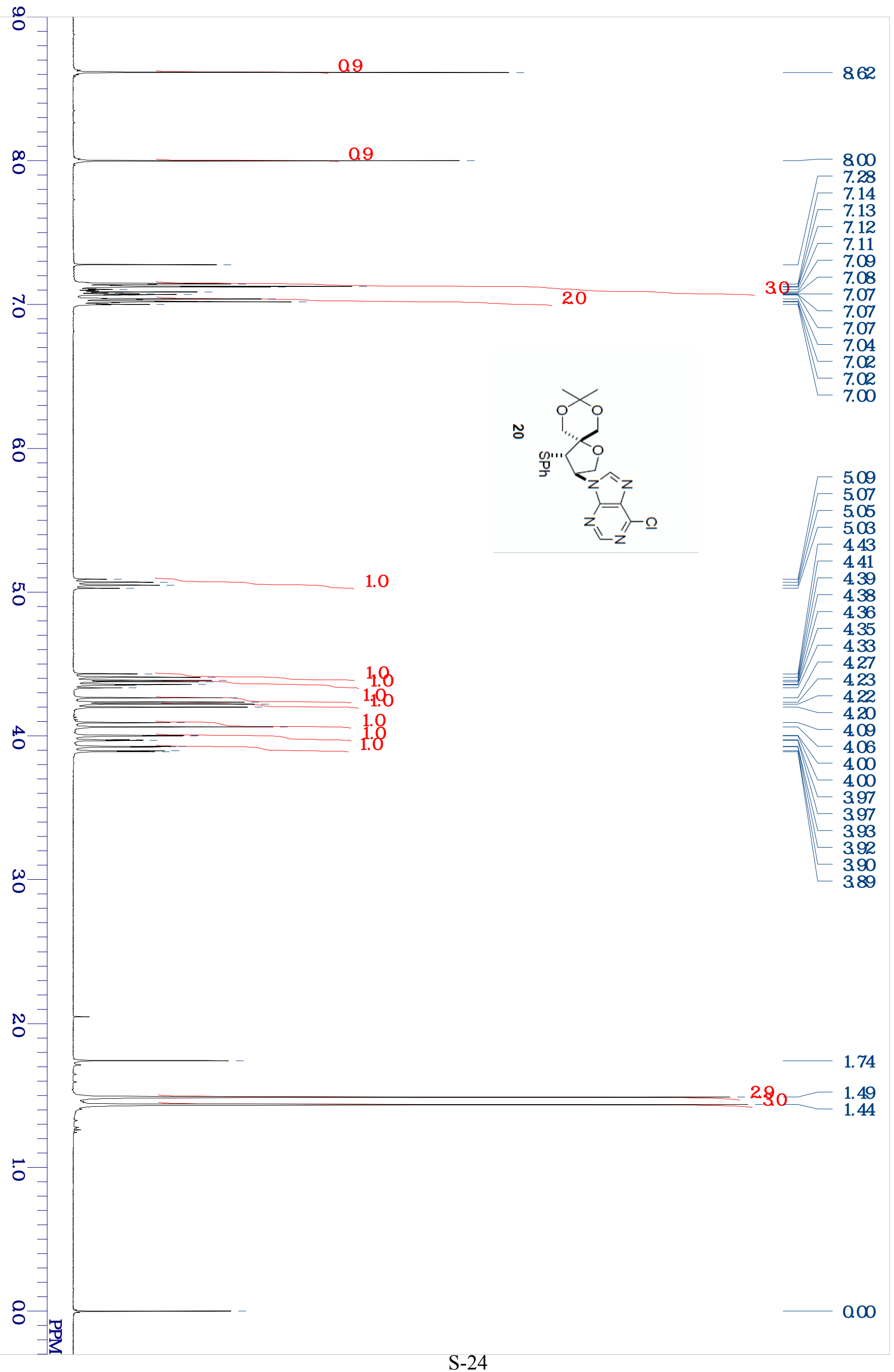




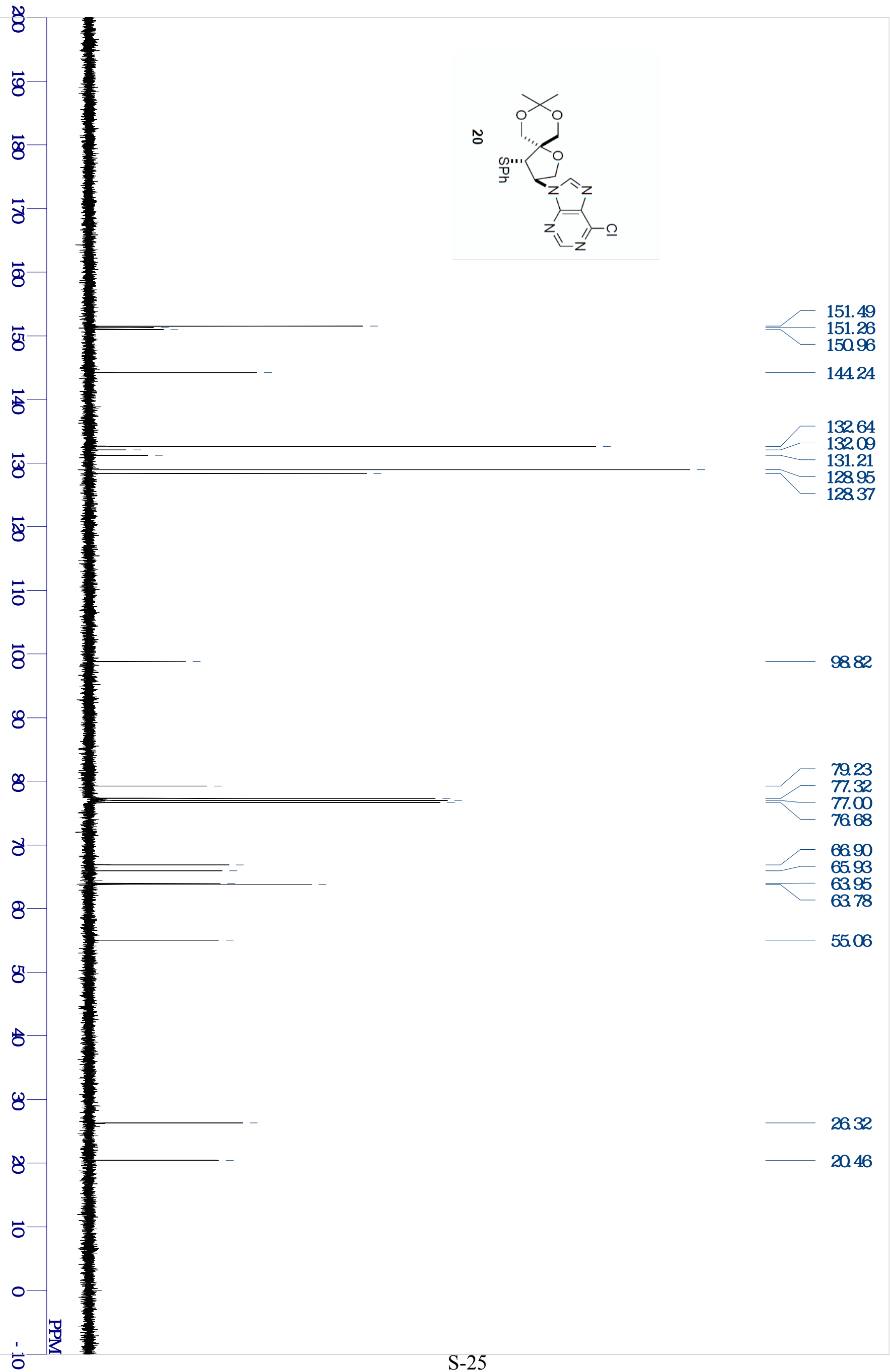




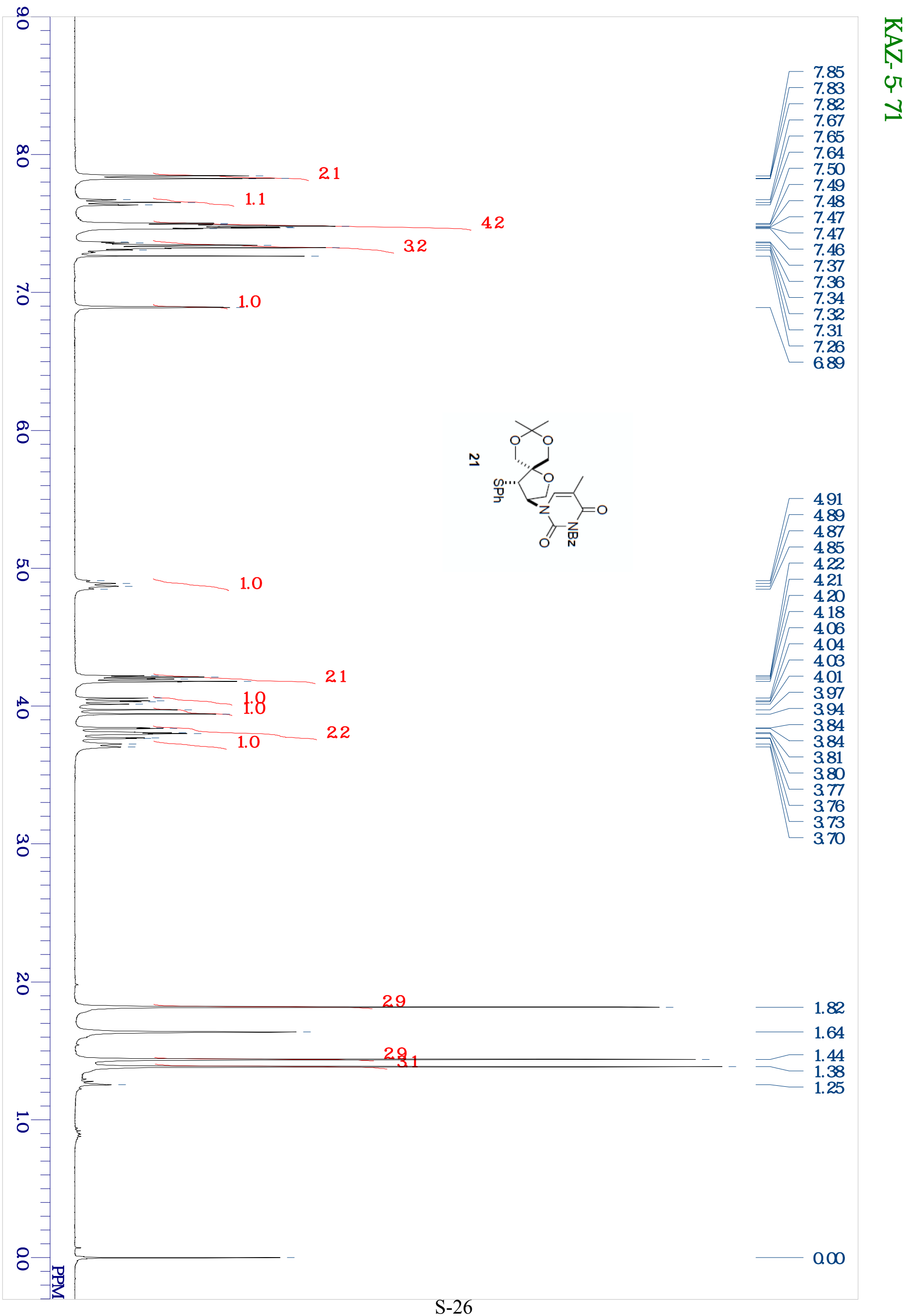




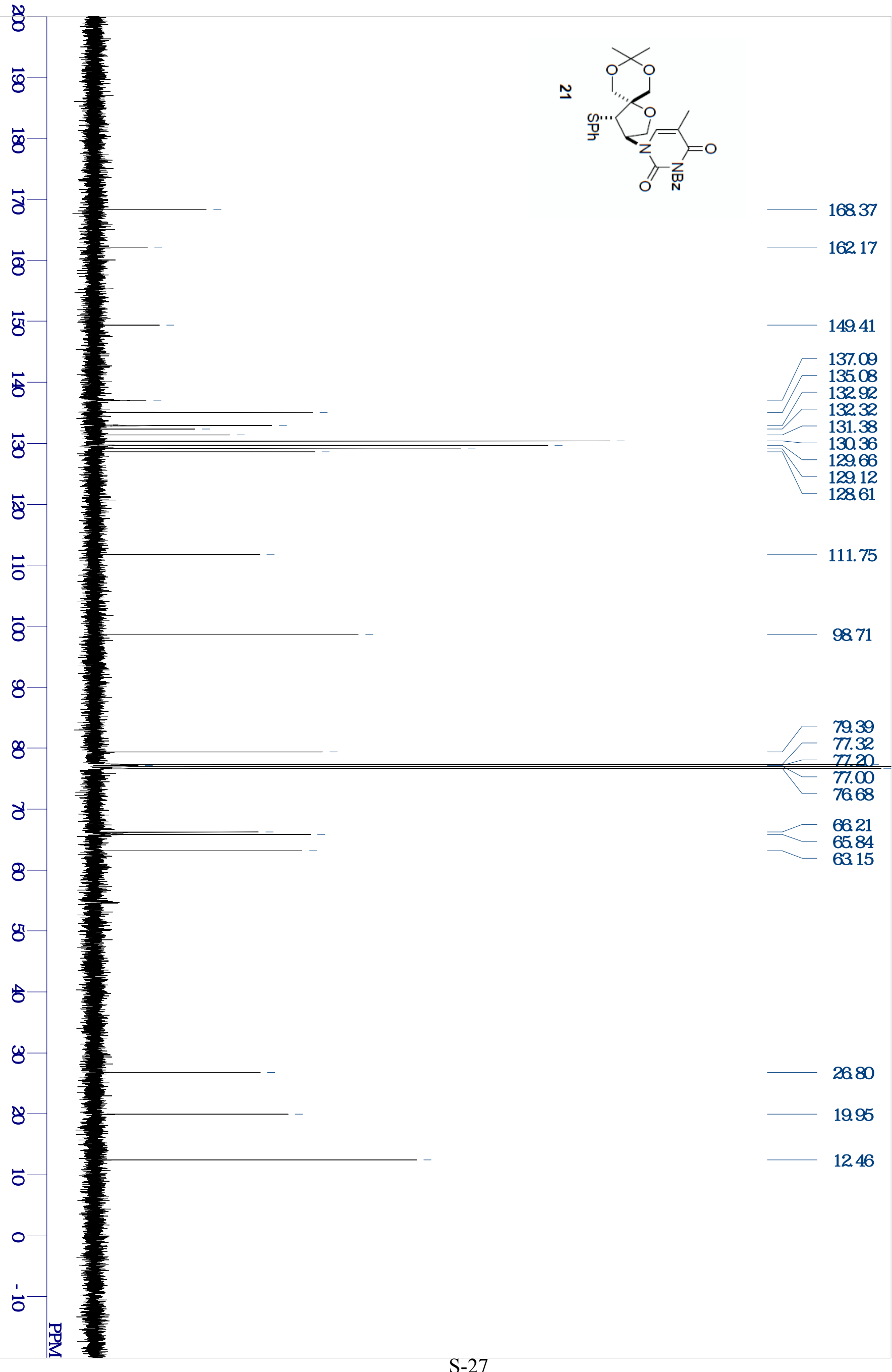




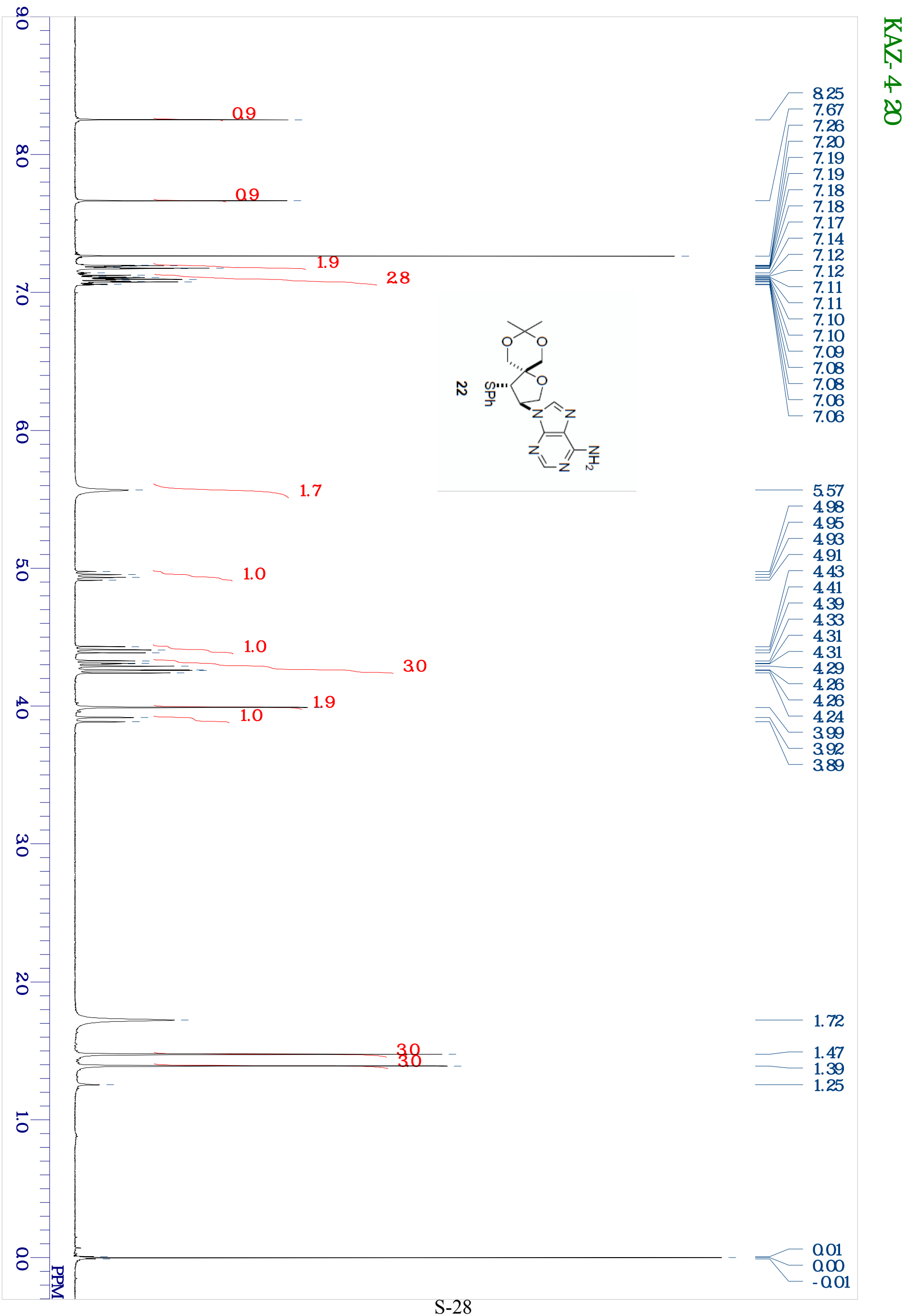




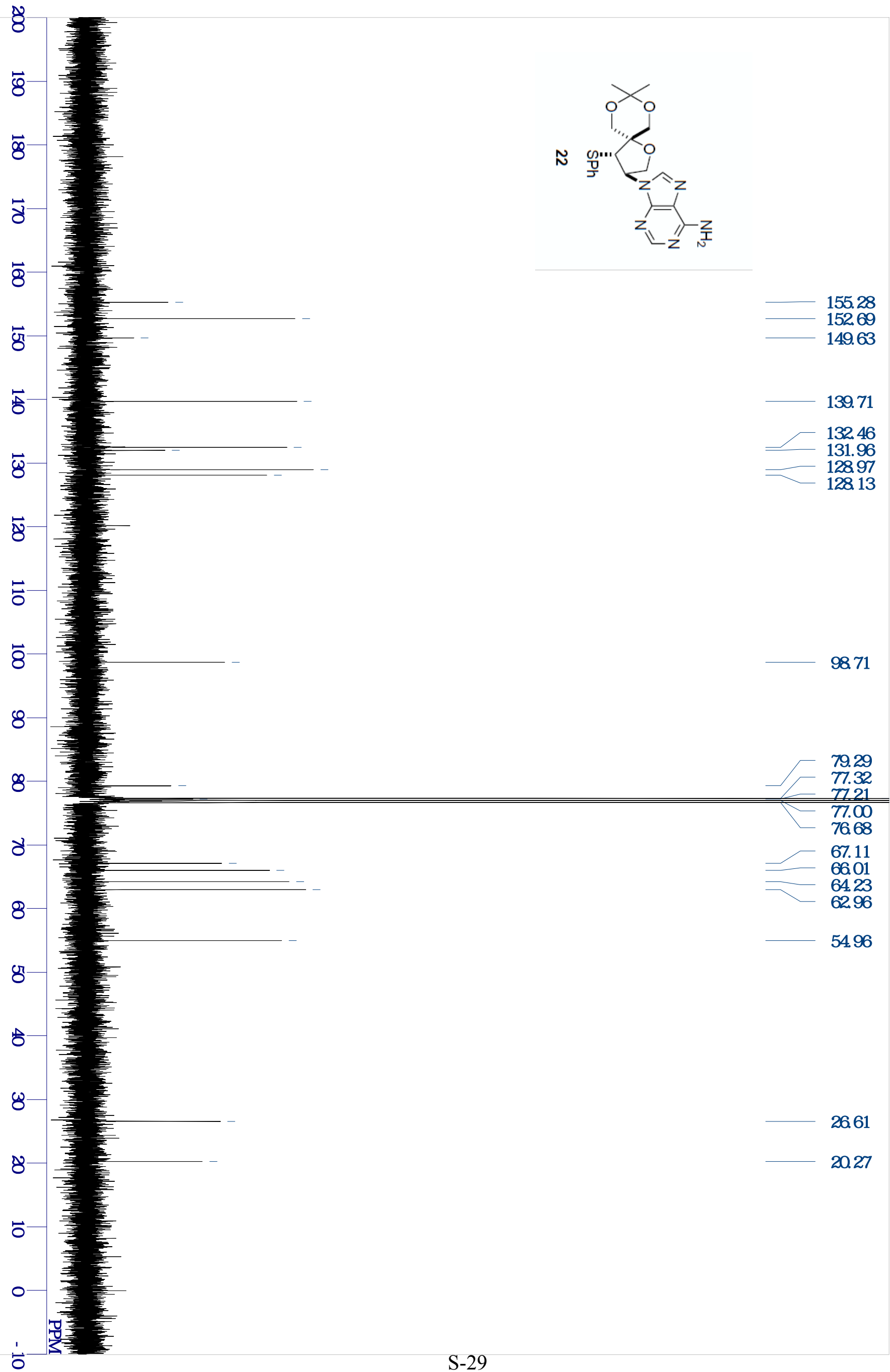




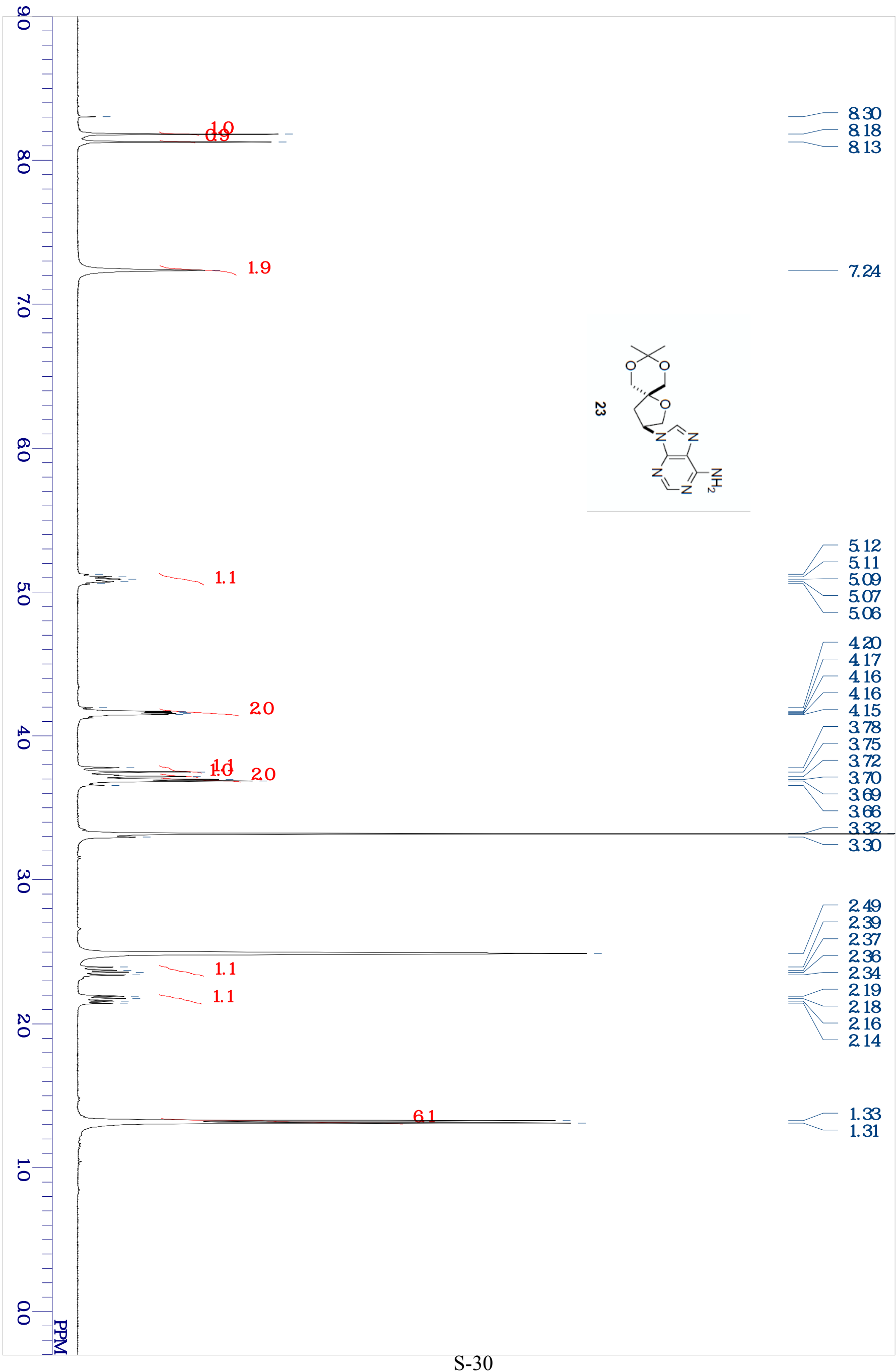




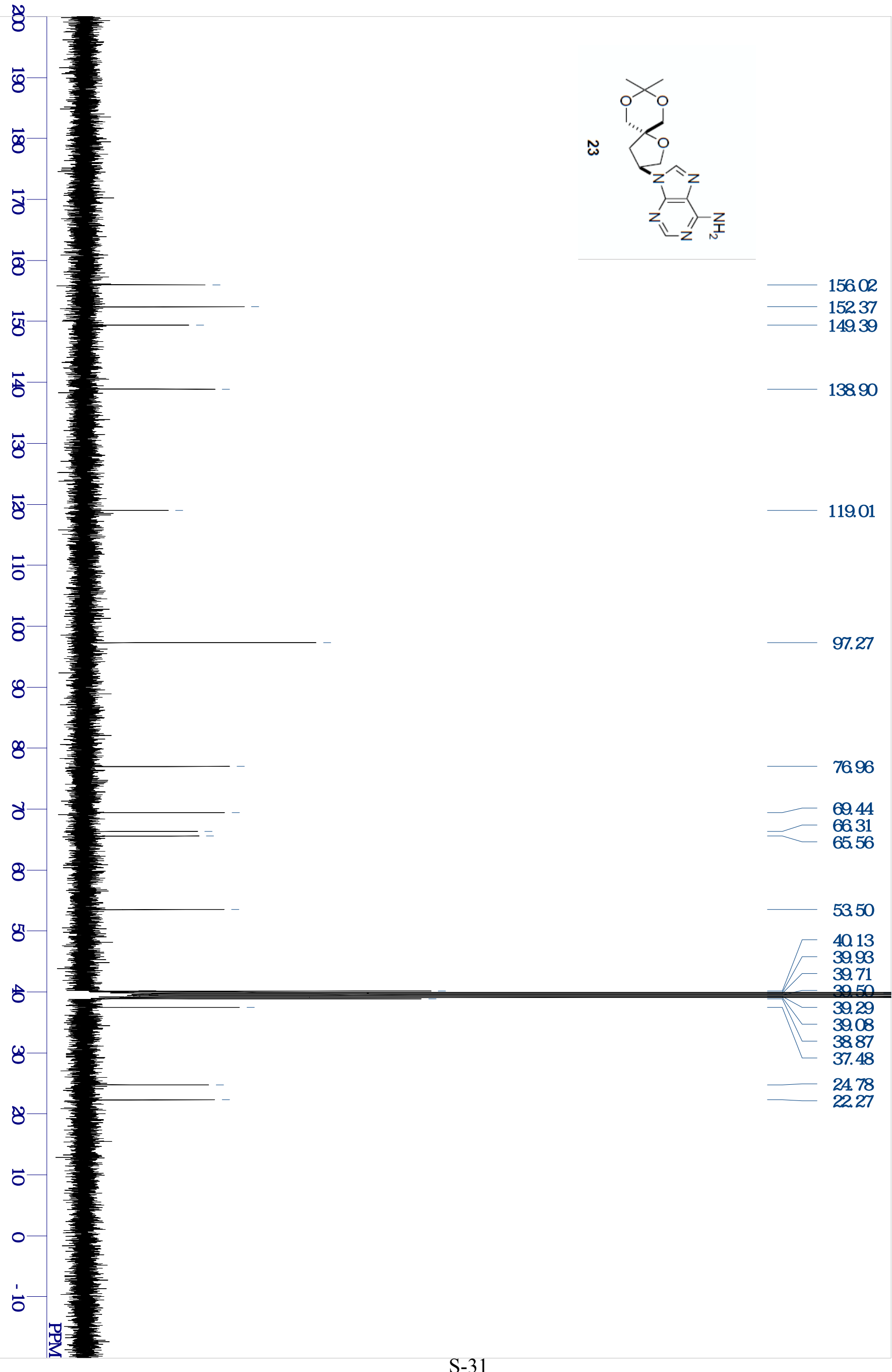




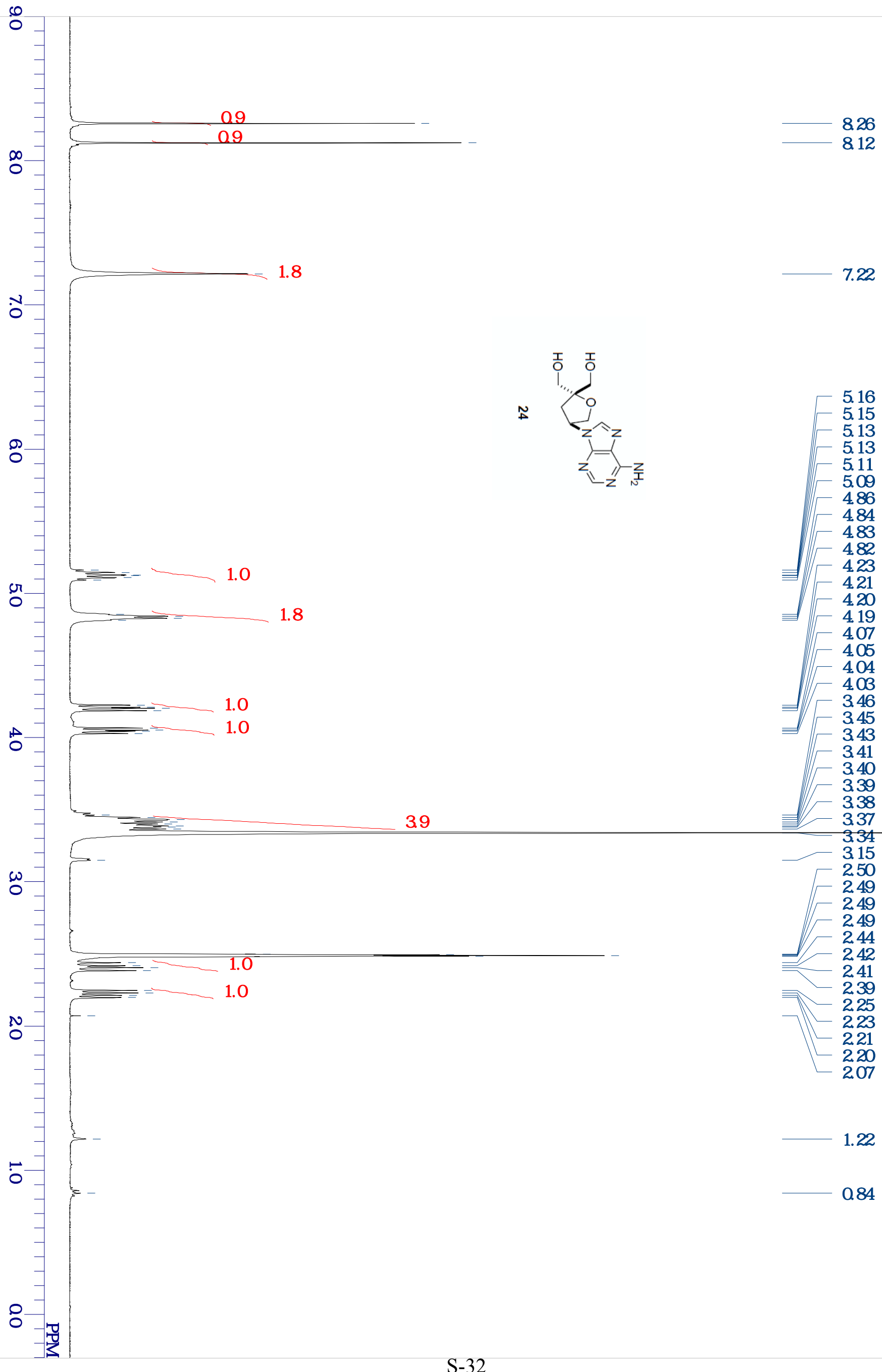




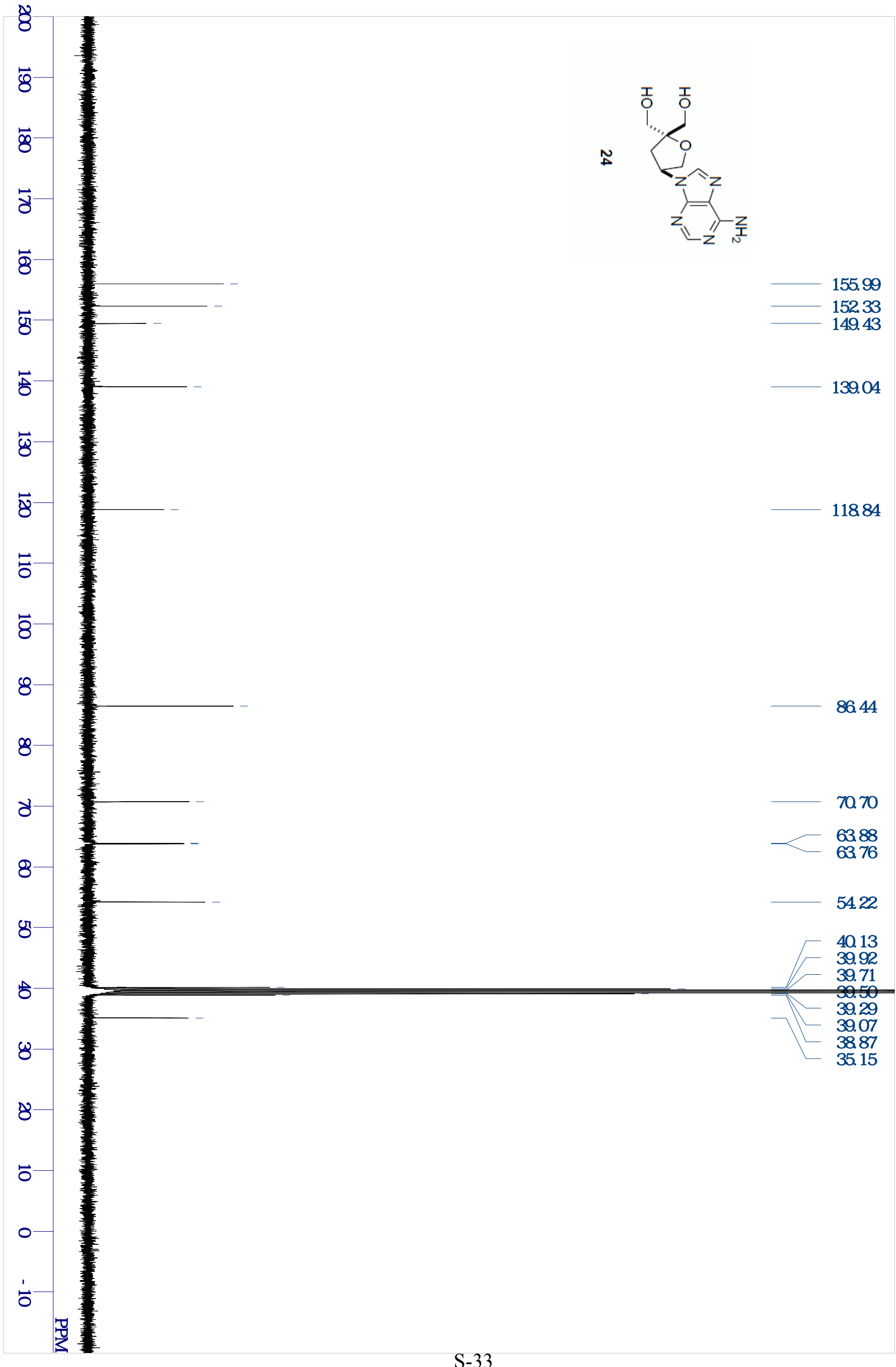




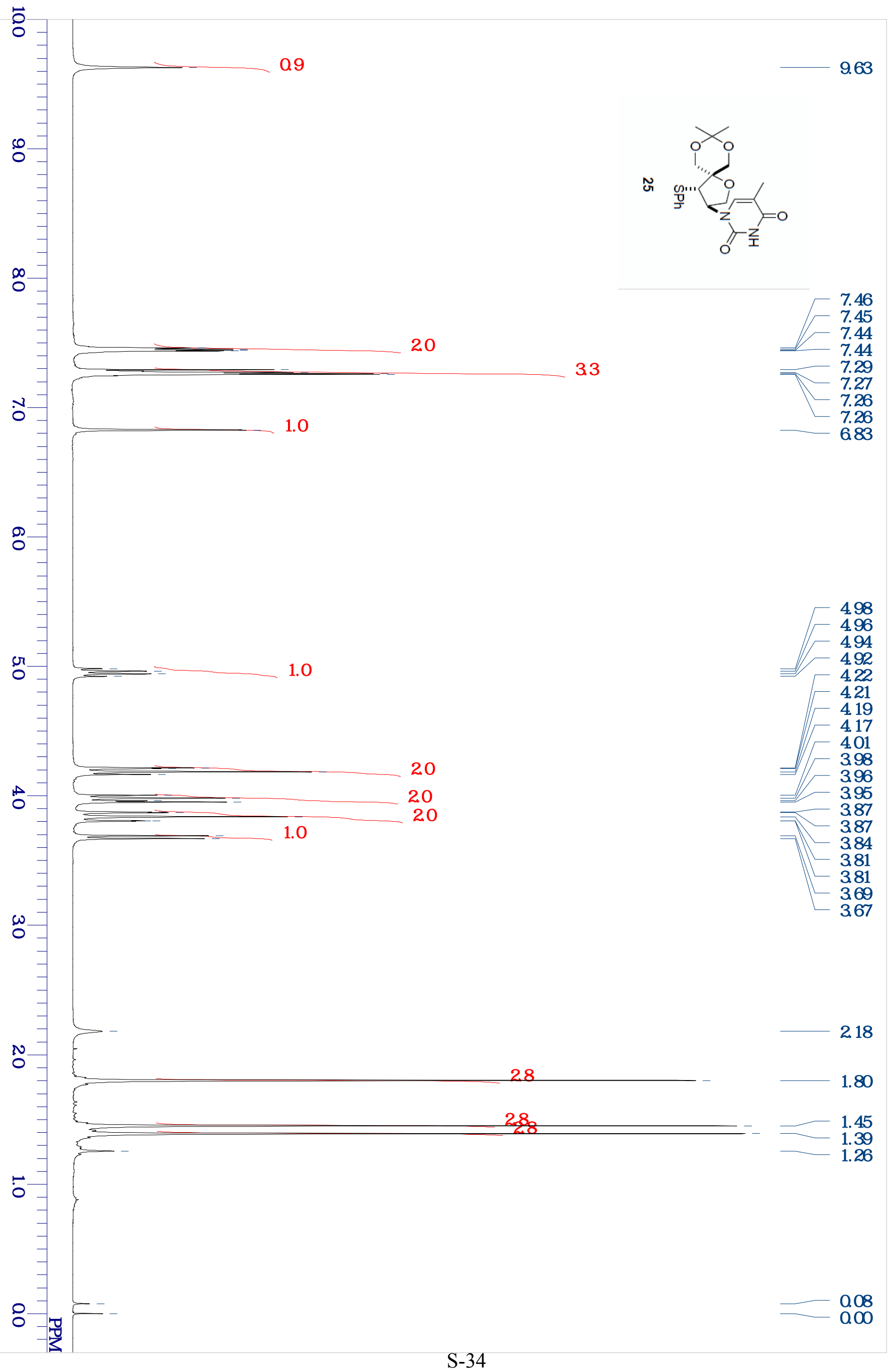




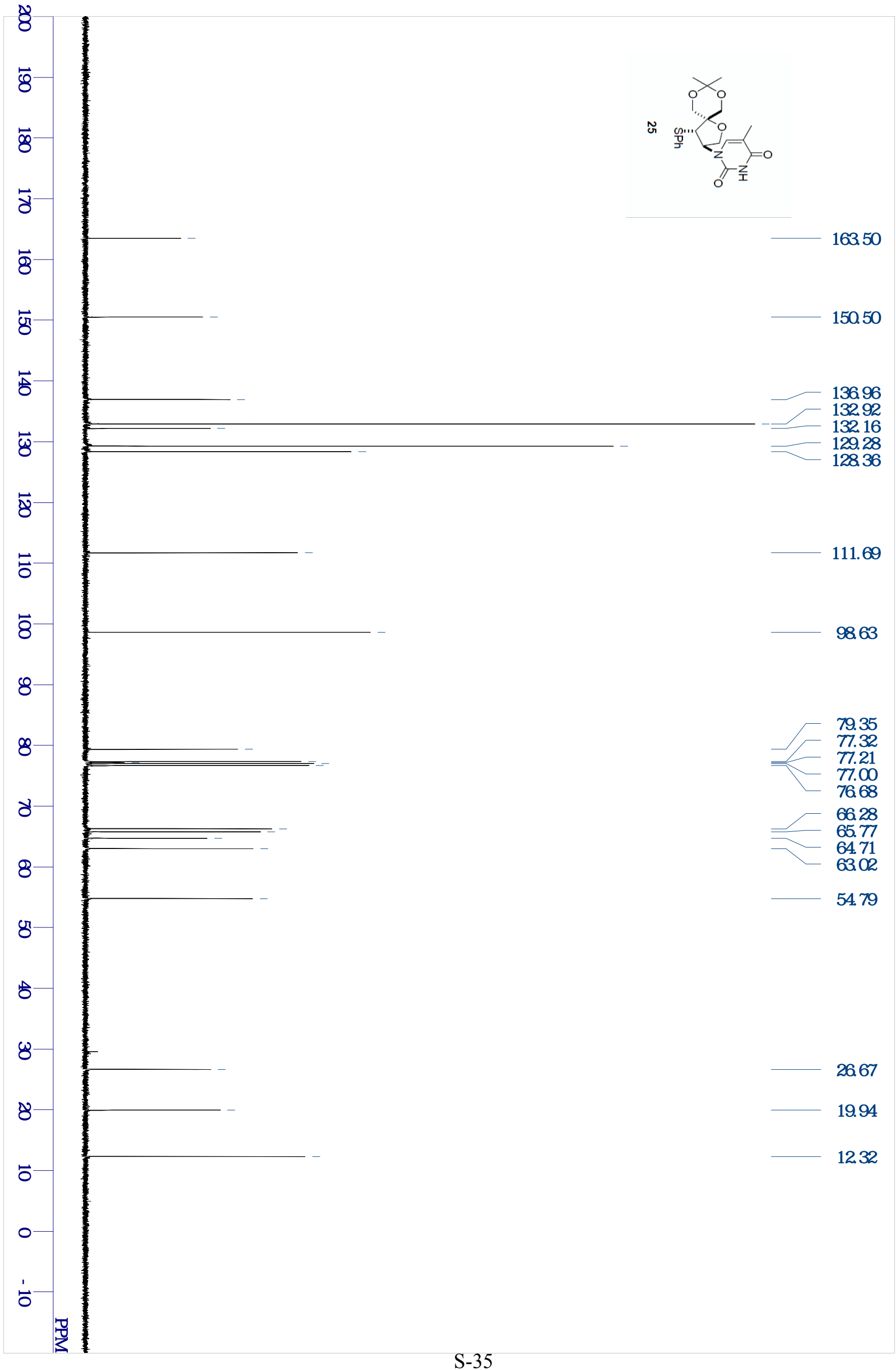




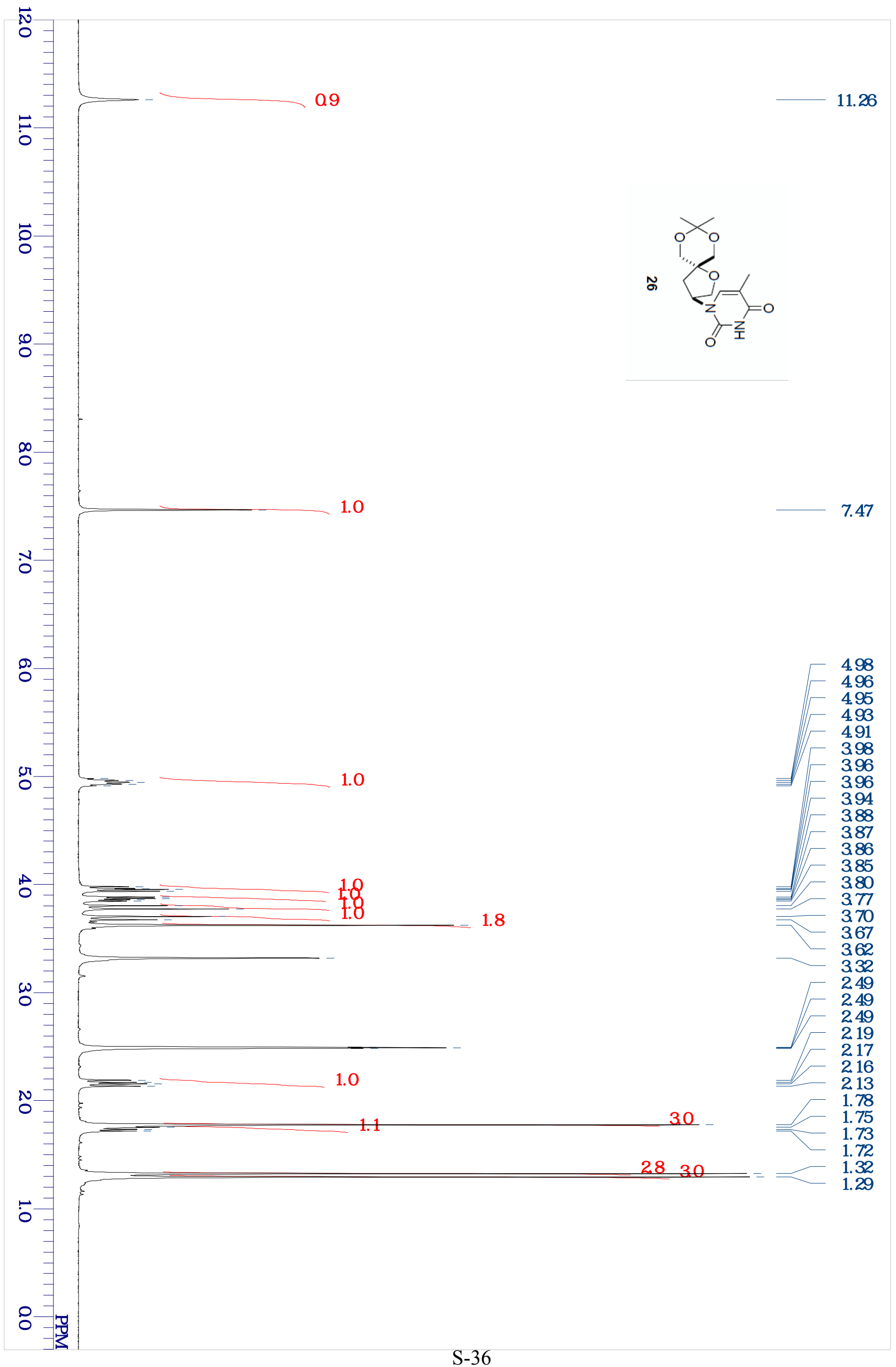




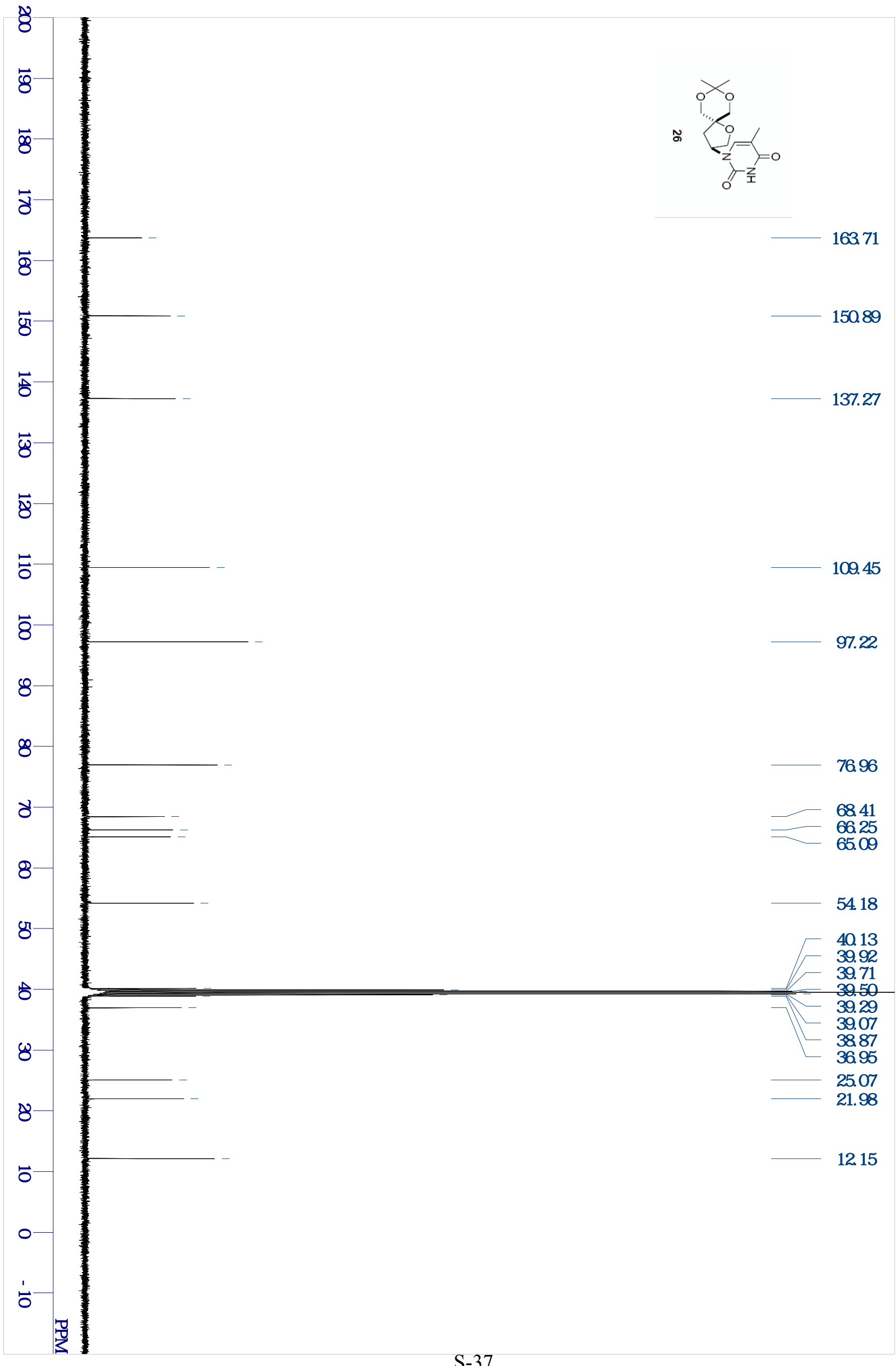




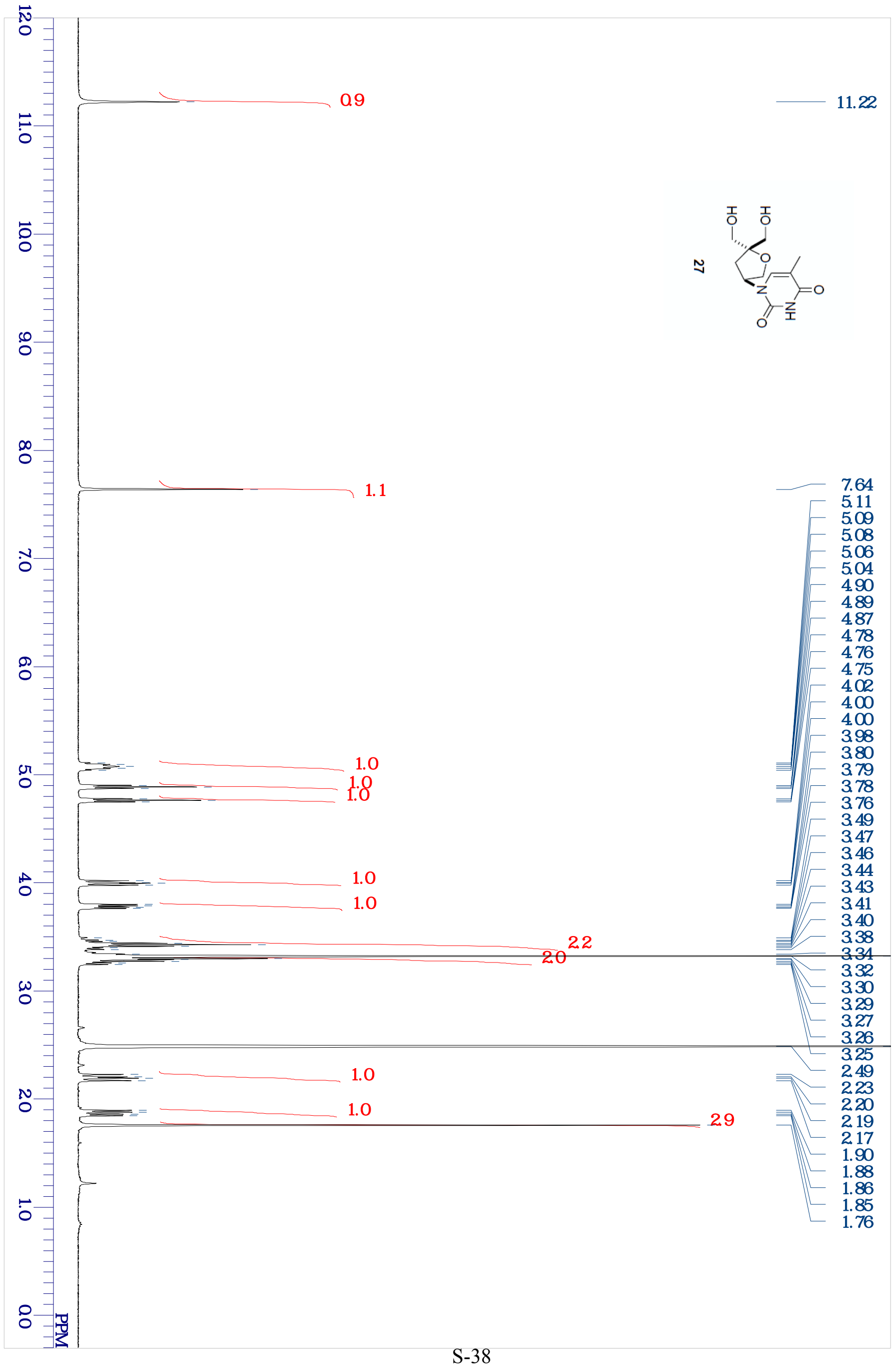




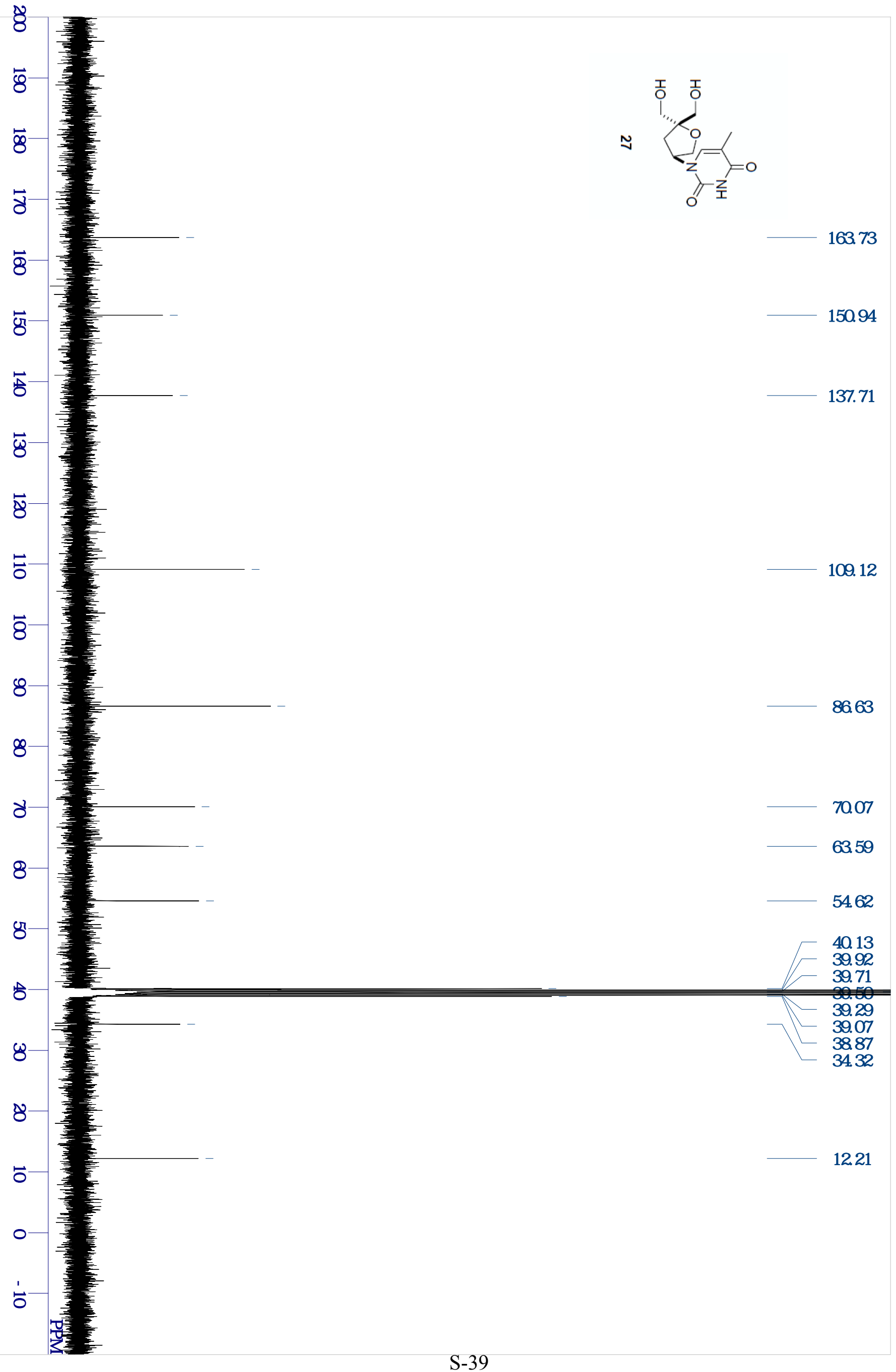

